\title{
Signal Set Design for Band-Limited Memoryless Multiple-Access Channels with Soft Decision Demodulation
}

\author{
PRAKASH NARAYAN, MEMBER, IEEE, AND DONALD L. SNYDER, FELLOW, IEEE
}

\begin{abstract}
Signal sets are identified that maximize the cutoff rate region for a multiple-access channel with an additive white Gaussian noise, in which the demodulator output alphaluet is allowed to be infinite ("infinitely soft decisions"). The optimizing designs consist of a simplex signal set for each sender, such that each sender's set is orthogonal to those of the other senders. For "second moment" and for "fractional out-of-bandenergy" bandwidth constraints on the signals of each sender, conditions are derived under which mutually orthogonal simplex sets are still optimal. For the second moment constraint, simplex sets derived from sinusoidal functions yield an optimal design and, for the out-of-band energy constraint, simplex sets derived from prolate spheroidal wave functions are optimal. Choices of signal sets that maximize the cutoff rate region for an additive shot-noise limited multiple-access optical channel, subject to average energy and peak amplitude constraints, are also identified.
\end{abstract}

\section{INTRODUCTION}

$\mathrm{I}^{\mathrm{r}}$ N A CODED digital communication system, the combination of the modulator, waveform channel, and demodulator can be considered to form a discrete channel for communication from the encoder to the decoder, which act to combat errors between a transmitted message and its reproduced version at the receiver. One would then like to design modulation schemes which would present the "best" discrete channel to the encoder and decoder, resulting in the optimum performance of the overall system. This is achieved by optimizing a suitable measure of performance of the discrete channel. Several parameters characterizing a single-user communication channel, such as channel capacity and error probability, have been proposed and used.

The use of the cutoff rate parameter $R_{0}$ as a criterion in evaluating the relative merits of different coding and modulation schemes was first proposed by Wozencraft and Kennedy [1] in 1966. In 1974, Massey [2] gave an eloquent argument in favor of the cutoff rate parameter $R_{0}$ as a measure of performance for the coordinated design of modulation and coding in a communication system. By

Manuscript recived Fcbruary 3, 1983; revised June 30, 1986. This work was supported in part by the National Science Foundation under Grants ECS-8113266 and ECS-8204449 and in part by the National Institutes of Health, Division of Research Resources, under Grant RR01380. This work was partially presented at the IEEE International Symposium on Information Theory, Santa Monica, CA, January 1981.

P. Narayan is with the Electrical Engineering Department, University of Maryland, College Park, MD 20742, USA.

D. L. Snyder is with the Department of Electrical Engineering, Washington University, St. Louis, MO 63130, USA.

IEEE Log Number 8612405 . interpreting $R_{0}$ as a function of the modulator and demodulator, Massey demonstrated how it could be used to design the best discrete channel as seen by the encoder and decoder. Although the "optimality" (with respect to the error probability criterion) of a simplex signal set on a single-user infinite-bandwidth additive white Gaussian noise channel had been conjectured for several years before 1974, it was not until then that Massey [2] rigorously demonstrated that such a signal set maximized the cutoff rate of this channel for infinitely soft decisions. To our knowledge, the simplex set has not yet been shown to optimize other performance measures such as channel capacity or the probability of a decoding error, nor has it been shown to be optimal under the $R_{0}$ criterion for other channels with Gaussian noise, such as a band-limited channel or a multiple-access channel. In this paper, we extend Massey's results to the multiple-access Gaussian channel by adopting the size of the cutoff rate region as the measure of goodness of the signal sets for the several senders. We show that mutually orthogonal simplex sets are the best choice of modulation when the senders are constrained only in their average energies. When the senders are also constrained in their bandwidth utilization, we establish conditions under which simplex sets remain optimal.

Snyder and Rhodes [3] identified modulation formats that maximize the cutoff rate parameter for a single-user shot-noise limited optical channel with infinite bandwidth under simultaneous constraints on average energy and peak amplitude. When the modulator uses up all the available energy, they showed that pulse-position modulation maximizes the cutoff rate $R_{0}$. We extend these results to two senders by maximizing the cutoff rate region and identifying the optimal signal sets. We also establish conditions under which this optimality is preserved when constraints are imposed on signal bandwidth.

A two-sender one-receiver multiple-access channel that is constant, discrete, and memoryless is specified by a set of transition probabilities

$$
p(w / u, v)
$$

where $u$ and $v$ are codeletters transmitted by sender 1 and sender 2 from their code alphabets $X_{1}=\{1, \cdots, a\}$ and $X_{2}=\{1, \cdots, b\}$, respectively, and where $w$ is a received 
letter in the alphabet $Y=\{1, \cdots, c\}$, in a manner that, for every positive integer $n$,

$$
P\left(\boldsymbol{y} / \boldsymbol{x}_{1}, \boldsymbol{x}_{2}\right)=\prod_{m=1}^{n} P\left(y_{m} / x_{1 m}, x_{2 m}\right)
$$

where $x_{1 m}, x_{2 m}$, and $y_{m}$ are the $m$ th digits transmitted by user 1 , transmitted by user 2 , and received over the channel, respectively.

We now assume that both senders employ block codes of length $n$ with $M_{1}$ and $M_{2}$ codewords, respectively, for sender 1 and sender 2 . Then the rate for sender $i$ is defined to be

$$
R_{i}=\frac{\ln M_{i}}{n} \text { nats } / \text { channel use }
$$

for $i=1,2$. Wc also assume that the encoders are fed by independent information sources so that the codeword $\boldsymbol{x}_{1}=\left(x_{11}, x_{12}, \cdots, x_{1 m}\right)$ transmitted by sender 1 is independent of the codeword $x_{2}=\left(x_{21}, x_{22}, \cdots, x_{2 m}\right)$ transmitted by sender 2 .

Consider now random coding for this two-user channel. Let $q_{1}$ and $q_{2}$ be probability distributions over the input alphabets $X_{1}$ and $X_{2}$, respectively, and let $C_{1}$ and $C_{2}$ be the particular codes employed by sender 1 and sender 2 , respectively. To each choice $\left(C_{1}, C_{2}\right)$, we assign the probability equal to the joint probability that one would pick $C_{1}$ when choosing each of the $n$ digits in the $M_{1}$ codewords independently according to $q_{1}$ and would pick $C_{2}$ when choosing each of the $n$ digits in the $M_{2}$ codewords independently according to $q_{2}$. Let $P_{e}$ denote the error probability for a particular code, and let $\bar{P}_{e}$ denote the average of $P_{e}$ for the above assignment of probabilities to the codes. We assume that an error occurs if the decoding decision for either sender 1 or sender 2 or both is in error.

Let $q$ denote a product distribution $q(u, v)=q_{1}(u) q_{2}(v)$ on $X_{1} \times X_{2}$, and let $\mathscr{R}_{0}(q)$ denote the region consisting of all rate pairs $\left(R_{1}, R_{2}\right)$ such that

$$
\begin{gathered}
0 \leq R_{1} \leq R_{1}^{*}(q) \\
0 \leq R_{2} \leq R_{2}^{*}(q) \\
R_{1}+R_{2} \leq R_{3}^{*}(q)
\end{gathered}
$$

where

$$
R_{1}^{*}(q)=-\ln \sum_{v} q_{2}(v) \exp \left\{-R_{1}^{*}\left(q_{1} / v\right)\right\}
$$

with

$$
R_{1}^{*}\left(q_{1} / v\right)=-\ln \sum_{w}\left[\sum_{u} q_{1}(u) p^{1 / 2}(w / u, v)\right]^{2}
$$

where $R_{2}^{*}(q)$ and $R_{2}^{*}\left(q_{2} / u\right)$ are similarly defined, and where

$$
R_{3}^{*}(q)=-\ln \sum_{w}\left[\sum_{u} \sum_{v} q(u, v) p^{1 / 2}(w / u, v)\right]^{2} .
$$

We shall call $R_{1}^{*}(q)$ the $q$-dependent cutoff rate of sender $l$ and shall call $R_{1}^{*}\left(q_{1} / v\right)$ the $q$-dependent cutoff rate of sender 1 given that sender 2 transmits the letter $v$. Similarly, we shall call $R_{3}^{*}(q)$ the $q$-dependent cutoff rate of the product channel. The region $\mathscr{R}_{0}(q)$ will be called the $q$-dependent cutoff region of the multiple-access channel itself. In [4], the cutoff region $\mathscr{R}_{0}$ of the multiple-access channel was defined as the convex hull of the union of the regions $\mathscr{R}_{0}(q)$ taken over all product distributions $q$.

Using the results of Slepian and Wolf [5], we argued in [4] that there exists a block code with the rate pair $\left(R_{1}, R_{2}\right)$ and codeword length $n$, such that the average probability of any decoding error $\bar{P}_{e}$ for maximum likelihood decoding is bounded according to

$$
\bar{P}_{e} \leq \sum_{i=1}^{3} \exp \left[-n\left\{R_{i}^{*}(q)-R_{i}\right\}\right]
$$

where

$$
\begin{aligned}
R_{i} & =R_{1}, & & \text { for } i=1 \\
& =R_{2}, & & \text { for } i=2 \\
& =R_{1}+R_{2}, & & \text { for } i=3
\end{aligned}
$$

for all rate pairs $\left(R_{1}, R_{2}\right)$ in the cutoff rate region. Thus, for block codes, the cutoff rate region provides a region of rates wherein the two senders can operate with an arbitrarily small error probability and also provides a measure of the coding complexity, as reflected by $n$, required to achieve this performance. In [4], we demonstrated that the senders need not have their frames synchronized for the validity of this assertion.

Peterson and Costello [6, theorem 1] have derived an upper bound on the average decoding error probability $\bar{P}_{e}$ for a two-sender one-receiver channel, using tree codes and maximum-likelihood decoding. Minimizing their bound as a function of $\rho_{i}$, where $0 \leq \rho_{i} \leq 1$ for $i=1,2,3$, using Gallager's method [7, p. 142], we obtain for rate pairs $\left(R_{1}, R_{2}\right)$ lying in the cutoff rate region

$$
\begin{aligned}
\vec{P}_{e} \leq & \exp \left\{-b T R_{1}^{*}(q)\right\} \cdot\left[\frac{\exp \left\{-b\left[R_{1}^{*}(q)-R_{1}\right]\right\}}{1-\exp \left\{-b\left[R_{1}^{*}(q)-R_{1}\right]\right\}}\right]+\exp \left\{-b T R_{2}^{*}(q)\right\} \cdot\left[\frac{\exp \left\{-b\left[R_{2}^{*}(q)-R_{2}\right]\right\}}{1-\exp \left\{-b\left[R_{2}^{*}(q)-R_{2}\right]\right\}}\right] \\
& +\exp \left\{-b T R_{3}^{*}(q)\right\} \cdot\left[\frac{\exp \left\{-b\left[R_{3}^{*}(q)-\left(R_{1}+R_{2}\right)\right]\right\}}{1-\exp \left\{-b\left[R_{3}^{*}(q)-\left(R_{1}+R_{2}\right)\right]\right\}}\right] \\
& \cdot\left[\frac{1+\exp \left\{-b\left[R_{1}^{*}(q)-R_{1}\right]\right\}}{1-\exp \left\{-b\left[R_{1}^{*}(q)-R_{1}\right]\right\}}+\frac{\exp \left(-b\left[R_{2}^{*}(q)-R_{2}\right]\right\}}{1-\exp \left\{-b\left[R_{2}^{*}(q)-R_{2}\right]\right\}}\right]
\end{aligned}
$$


where $b$ is the number of codeletters assigned to each branch of the tree and $T$ is the length of the tail of the tree [6]. The constraint length of the tree code is defined to be $b T$. Equation (9) can be used to obtain estimates of $b$ and $T$ needed to achieve a specified level of performance with tree codes.

It is evident from (8) and (9) that for both block and tree coding, the cutoff rate region describes a region of rate pairs wherein one can operate with an arbitrarily small error probability. Moreover, (8) and (9) also provide conservative estimates of the coding complexity needed to achieve a specified level of performance for rate pairs in the cutoff rate region.

The cutoff rate region for a given communication system will depend on the modulation formats adopted by the two senders. Here, the optimal modulation scheme is considered to be the one that maximizes the cutoff rate region in some sense. It is desirable that the cutoff rate region be "large" so that high code rates can be accommodated; or so that, for a given rate pair, the bounds in (8) and (9) on the error probabilities are small, or so that, for a given rate pair, the coding complexity sufficient to achieve a specified reliability is small.

The bandwidth constraints adopted by us are described in Section II. The problem of "maximizing" the cutoff rate region for an additive-signal additive white Gaussian noise channel with a bandwidth limitation is examined in Section III. In Section IV, an additive-signal shot-noise limited channel with bandwidth constraints is treated.

\section{BANDWIDTH CONSTRAINTS}

Either by virtue of law or nature, it is generally necessary to impose constraints on the portion of the frequency spectrum that the signals transmitted by a sender can occupy. There is no universal measure of bandwidth for a signal $s(t)$ of finite time duration. Several measures have been used [8], [9], and we consider two of these below.

\section{A. Second-Moment Bandwidth Constraint}

Let $S(f),-\infty<f<\infty$, be the "spectrum" or the Fourier transform of the signal $s(t), t \in[0, T]$, where

$$
S(f)=\int_{-\infty}^{\infty} s(t) \exp (-j 2 \pi f t) d t .
$$

The signal $s(t)$ can be defined to have a bandwidth less than or equal to $B$ if

$$
\left(\int_{-\infty}^{\infty} f^{2}|S(f)|^{2} d f\right) /\left(\int_{-\infty}^{\infty}|S(f)|^{2} d f\right) \leq B^{2}
$$

where $|S(f)|$ denotes the magnitude of $S(f)$. The inequality (11) is referred to as the "second-moment bandwidth constraint" as it imposes a constraint on the second moment of the spectrum of $s(t)$.

Using Parseval's theorem, it is seen that (11) can also be expressed as

$$
\left(\int_{-\infty}^{\infty} \dot{s}^{2}(t) d t\right) /\left(\int_{-\infty}^{\infty} s^{2}(t) d t\right) \leq B^{2}
$$

where $\dot{s}(t)$ denotes the time derivative of $s(t)$. If the energy in the signal $s(t)$ is fixed, then (12) constrains the energy in its time derivative. This ensures that $s(t)$ is a "smooth" function of $t$.

\section{B. Out-of-Band Energy Constraint}

An alternative way to define the bandwidth of a signal $s(t)$ is to limit the fraction of its energy lying outside the frequency band $[-B, B]$. The signal energy contained in this band is

$$
\int_{-B}^{B}|S(f)|^{2} d f
$$

and its total energy is

$$
\int_{-\infty}^{\infty}|S(f)|^{2} d f
$$

where $S(f)$ is given by (10). For some specified $\epsilon, 0<\epsilon<1$, one can then define the signal to have a bandwidth less than or equal to $B$ if

$$
\left(\int_{-B}^{B}|S(f)|^{2} d f\right) /\left(\int_{-\infty}^{\infty}|S(f)|^{2} d f\right) \geq 1-\epsilon .
$$

Note that $\epsilon$ is the maximum fraction of energy in $s(t)$ allowed to fall outside the frequency band $-B \leq f \leq B$.

\section{Additive Signals, Additive White Gaussian Noise Channel}

The model is shown in Fig. 1. The modulators produce finite energy signals from the sets $\mathscr{G}=\{g(t ; i) ; i=$ $1,2, \cdots, a\}$ and $\mathscr{H}=\{h(t ; l) ; l=1,2, \cdots, b\}$ for senders 1 and 2 , respectively, where $g(t ; i)$ is transmitted by sender 1 if his encoder produces codeletter $i$, and $h(t ; l)$ is transmitted by sender 2 if his encoder produces codeletter $l$. Signals are transmitted and received on the interval $[0, T]$. The signal $r(t)$ received at the output of the channel is

$$
r(t)=g(t ; i)+h(t ; l)+n(t), \quad 0 \leq t \leq T
$$

where $n(t)$ is white Gaussian noise with a two-sided power spectral density of $N_{0} / 2 \mathrm{~W} / \mathrm{Hz}$. The demodulator produces an output $w$, from the alphabet $Y=\{1, \cdots, c\}$.

From a coding viewpoint, the modulators, waveform channel, and demodulator together constitute a discrete channel. By virtue of the stationarity and the independent increments property of the white Gaussian noise process, this is a constant discrete memoryless channel in the sense of (2).

The cutoff rate region of any channel is not decreased by using a finer output quantization. Thus we initially examine the limiting situation when the output quantization is arbitrarily fine, that is, $c=\infty$. The effect of a finite quantization for our model will follow directly from the corresponding result of Massey [2]. 


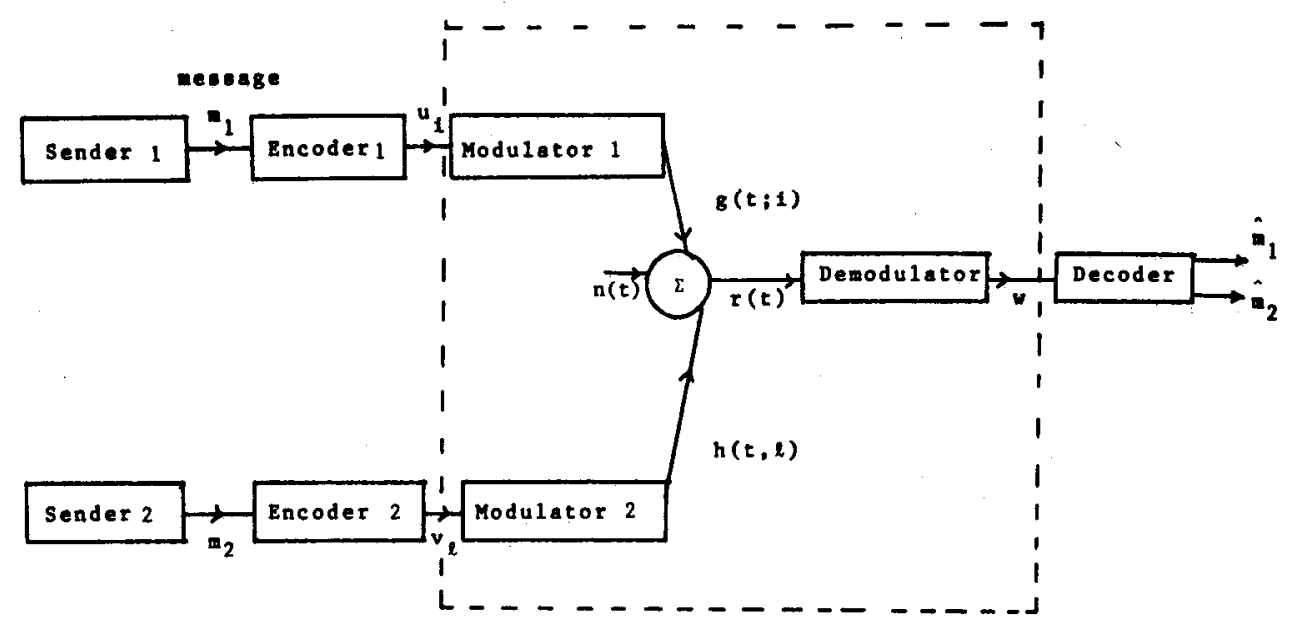

Fig. 1. Model of two-sender one-receiver additive white Gaussian noise channel.

When the demodulator output is unquantized, we demonstrate in Appendix I that (4)-(7) become

$$
\begin{gathered}
R_{1 \infty}^{*}(q)=-\ln \sum_{i, j=1}^{a} q_{1}\left(u_{i}\right) q_{1}\left(u_{j}\right) \exp \left(-\xi_{i j} / 4 N_{0}\right) \\
R_{2 \infty}^{*}(q)=-\ln \sum_{l, k=1}^{b} q_{2}\left(v_{l}\right) q_{2}\left(v_{k}\right) \exp \left(-\eta_{l k} / 4 N_{0}\right) \\
R_{3 \infty}^{*}(q)=-\ln \sum_{i, j=1}^{a} \sum_{l, k=1}^{b} q_{1}\left(u_{i}\right) q_{1}\left(u_{j}\right) q_{2}\left(v_{l}\right) q_{2}\left(v_{k}\right) \\
\cdot \exp \left(-\gamma_{i j l k} / 4 N_{0}\right)
\end{gathered}
$$

where $R_{1 \infty}^{*}(q), R_{2 \infty}^{*}(q)$, and $R_{3 \infty}^{*}(q)$ denote, respectively, the values of $R_{1}^{*}(q), R_{2}^{*}(q)$, and $R_{3}^{*}(q)$ for $c=\infty$, and where

$$
\begin{aligned}
\xi_{i j} & =\int_{0}^{T}\{g(t ; i)-g(t ; j)\}^{2} d t, \\
\eta_{l k} & =\int_{0}^{T}\{h(t ; l)-h(t ; k)\}^{2} d t,
\end{aligned}
$$

and

$\gamma_{i j / k}=\int_{0}^{T}\{g(t ; i)-g(t ; j)+h(t ; l)-h(t ; k)\}^{2} d t$.

By defining

$$
A=\sum_{i=1}^{a} q_{1}^{2}\left(u_{i}\right)
$$

and by using Jensen's inequality, Massey [2] has shown that the right side of (15) can be bounded above as

$$
\begin{array}{r}
R_{1 \infty}^{*}(q) \leq-\ln \left[A+(1-A) \exp \left\{-4 N_{0}(1-A)^{-1}\right.\right. \\
\left.\left.\cdot \sum_{i, j=1}^{a} q_{1}\left(u_{i}\right) q_{1}\left(u_{j}\right) \xi_{i j}\right\}\right],
\end{array}
$$

with equality holding if and only if $\xi_{i j}$ has the same value whenever $i \neq j$. The right side of (22) is a monotonically increasing function of $\xi_{i j}, i \neq j$. Thus if $\xi$ denotes the maximum of $\xi_{i j}$, we obtain

$$
R_{1 \infty}^{*}(q) \leq-\ln \left[A+(1-A) \exp \left(-\xi / 4 N_{0}\right)\right]
$$

with equality holding if and only if $\xi_{i j}=\xi$ whenever $i \neq j$. Similarly, letting

$$
B=\sum_{l=1}^{b} q_{2}^{2}\left(v_{l}\right)
$$

we find that

$$
R_{2 \infty}^{*}(q) \leq-\ln \left[B+(1-B) \exp \left(-\eta / 4 N_{0}\right)\right]
$$

where $\eta$ denotes the maximum of $\eta_{l k}$ and where equality holds in (25) if and only if $\eta_{l k}=\eta$ whenever $l \neq k$.

If $\gamma$ denotes the maximum of $\gamma_{i j l k}$ for $i \neq j$ and $l \neq k$, it follows similarly that

$$
\begin{aligned}
R_{3 \infty}^{*}(q) \leq & -\ln \left[A B+B(1-A) \exp \left(-\xi / 4 N_{0}\right)\right. \\
& +A(1-B) \exp \left(-\eta / 4 N_{0}\right) \\
& \left.+(1-A)(1-B) \exp \left(-\gamma / 4 N_{0}\right)\right]
\end{aligned}
$$

with equality holding if and only if, in addition to $\xi_{i j}$ and $\eta_{l k}$ being equal to $\xi$, for $i \neq j$, and $\eta$, for $l \neq k$, it also holds that $\gamma_{i j l k}=\gamma$ whenever $i \neq j$ and $l \neq k$.

A simple proof given in Appendix II shows that for all $i \neq j$ and $l \neq k$, a necessary and sufficient condition for $\gamma_{i j l k}$ to be constant when $\xi_{i j}$ and $\eta_{l k}$ are constant is that the signal sets $\mathscr{G}=\{g(t ; i): i=1,2, \cdots, a\}$ and $\mathscr{H}=$ $\{h(t ; l): l=1,2, \cdots, b\}$ of the two senders lie in mutually orthogonal spaces. Thus, if (26) holds with equality, so also do (23) and (25) with

$$
\gamma=\xi+\eta
$$

In this case, it follows from (26) and (27) that

$$
R_{3 \infty}^{*}(q)=R_{1 \infty}^{*}(q)+R_{2 \infty}^{*}(q) \text {. }
$$

Hence, in the definition of the cutoff rate region (4)-(7), the condition (4c) on the sum of the rates becomes redundant by virtue of (28). Furthermore, we see from (21) that the right side of (23) depends on the codeletter probability distribution $q_{1}$ for the first sender but not on the codeletter probability distribution $q_{2}$ for the second sender. 
Similarly, the right side of (25) is a function of $q_{2}$ but not of $q_{1}$. The maximizing distributions for the right sides of (23) and (25) are easily seen to be the uniform probability distributions

$$
q_{1}\left(u_{i}\right)=a^{-1}
$$

for $i=1, \cdots, a$, and

$$
q_{2}\left(v_{l}\right)=b^{-1}
$$

for $l=1, \cdots, b$. Consequently, we obtain from (21) that $A=a^{-1}, B=b^{-1}$. It then easily follows that an outer bound on the cutoff rate region is the region of all rate pairs $\left(R_{1}, R_{2}\right)$ satisfying the simplified conditions

$$
R_{1} \leq-\ln \left[a^{-1}+\left(1-a^{-1}\right) \exp \left(-\xi / 4 N_{0}\right)\right]
$$

and

$$
R_{2} \leq-\ln \left[b^{-1}+\left(1-b^{-1}\right) \exp \left(-\eta^{2} / 4 N_{0}\right)\right]
$$

where $\xi$ and $\eta$ are the allowed maxima of $\xi_{i j}$ and $\eta_{i j}$, respectively. Moreover, the actual cutoff rate region coincides with this outer bound if and only if the two users' signal sets can be chosen as mutually orthogonal simplexes with $\xi_{i j}=\xi$ for $i \neq j$, and $\eta_{l k}=\eta$ for $l \neq k$. That the signal sets achieving the maximal outer bounds in (31) are simplex sets [10, pp. 259-261], follows from Massey [2] and is easily seen as follows. Rewriting $\xi$ as

$$
\xi=\int_{0}^{T}[g(t ; i)-g(t ; j)]^{2} d t
$$

for $i \neq j$, we obtain upon summation over $i \neq j$ that

$$
\begin{aligned}
\xi=2(a-1) \begin{array}{l}
1 \\
{\left[\sum_{i=1}^{a} \int_{0}^{T}\{g(t ; i)\}^{2} d t\right.}
\end{array} & \left.-\frac{1}{a} \int_{0}^{T}\left\{\sum_{i=1}^{a} g(t ; i)\right\}^{2} d t\right] .
\end{aligned}
$$

Clearly, $\xi$ is maximized when the second term on the right side of (33) is zero, i.e., when the signal set of the first sender achieving the outer bound in (31) is also a simplex. The same is true of the signal set of the second sender achieving the maximal outer bound in (31). The corresponding maximum values of $\xi$ and $\eta$ are given by

$$
\begin{aligned}
& \xi=2(a-1)^{-1} \sum_{i=1}^{a} \int_{0}^{T}\{g(t ; i)\}^{2} d t \\
& \eta=2(b-1)^{-1} \sum_{i=1}^{b} \int_{0}^{T}\{h(t ; l)\}^{2} d t .
\end{aligned}
$$

If we now constrain the average energies of the signals in $\mathscr{G}$ and $\mathscr{H}$ to be equal to $E_{1}$ and $E_{2}$, respectively, i.e., if

$$
a^{-1} \sum_{l=1}^{b} \int_{0}^{T}\{g(t ; i)\}^{2} d t=E_{1}
$$

and

$$
a^{-1} \sum_{l=1}^{b} \int_{0}^{T}\{h(t ; l)\}^{2} d t=E_{2},
$$

then from (31) and (34)-(37) it follows that an outer bound on the cutoff rate region is the region of all rate pairs $\left(R_{1}, R_{2}\right)$ satisfying

$$
R_{1} \leq-\ln \left[a^{-1}+\left(1-a^{-1}\right) \exp \left\{-a E_{1} / 2 N_{0}(a-1)\right\}\right]
$$

and

$$
R_{2} \leq-\ln \left[b^{-1}+\left(1-b^{-1}\right) \exp \left\{-b E_{2} / 2 N_{0}(b-1)\right\}\right] .
$$

It remains to be shown when, with the given energy constraints in (36) and (37) and with bandwidth constraints, there exist mutually orthogonal simplex sets which achieve the outer bounds in (38), with $\xi$ and $\eta$ taking on the maximum values allowed by the constraints. Specifically, we shall identify signal sets with minimum bandwidths (in the sense of (12) and (13)) which achieve these outer bounds.

\section{A. Signal Sets with Minimum Second Moment Bandwidths}

We define the squared second moment bandwidths $B_{1}^{2}$ and $B_{2}^{2}$ (see (12)) of the signals in $\mathscr{G}=\{g(t ; i): i=$ $1, \cdots, a\}$ and $\mathscr{H}=\{h(t ; l): l=1, \cdots, b\}$, respectively, as

$$
B_{1}^{2}(\mathscr{G})=\frac{1}{a E_{1}} \sum_{i=1}^{a} \int_{0}^{T}\{\dot{g}(t ; i)\}^{2} d t
$$

and

$$
B_{2}^{2}(\mathscr{H})=\frac{1}{b E_{2}} \sum_{l=1}^{b} \int_{0}^{T}\{\dot{h}(t ; l)\}^{2} d t
$$

where $\dot{g}(t ; i)$ and $\dot{h}(t ; l)$ represent time derivatives of $g(t ; i)$ and $h(t ; l)$. Our aim is to identify signal sets with minimal bandwidths, as defined by (39) and (40), which achieve the outer bounds in (38).

This leads us to the following optimization problem: select signal sets $\mathscr{G}=\{g(t ; i): i=1, \cdots, a\}$ and $\mathscr{H}=$ $\{h(t ; l): l=1, \cdots, b\}$ to minimize

$$
\dot{J}_{1}=\sum_{i=1}^{a} \int_{0}^{T}\{\dot{g}(t ; i)\}^{2} d t
$$

and

$$
J_{2}=\sum_{l=1}^{b} \int_{0}^{T}\{\dot{h}(t ; l)\}^{2} d t
$$

subject to the average energy constraints (36), (37), and the constraint that $\mathscr{G}$ and $\mathscr{H}$ are mutually orthogonal simplex sets. Because we are restricting ourselves to simplex signal sets, it follows that (36) is met in the following manner:

$$
\int_{0}^{T}\{g(t ; i)\}^{2} d t=E_{1}
$$

for $i=1, \cdots, a$. A similar statement holds for (37). We shall present below the minimization of (41). The minimization of (42), which is identical, will be omitted; only the results will be stated whenever appropriate.

The existence of the integrals in (41) requires the continuity at $t=0$ and at $t=T$ of $g(t ; i)$ for $i=1, \cdots, a$. This gives rise to the following boundary conditions:

$$
g(0 ; i)=g(T ; i)=0
$$

for $i=1, \cdots, a$. 
The minimization problem is simplified by temporarily ignoring the constraint of mutually orthogonal simplex sets in obtaining the solutions. It will be seen that solutions to this relaxed prohlem can he used to construct solutions to the fully constrained problem.

We first minimize $J_{1}$, given by (41), subject to the constraint (43) and to the boundary conditions (44). Consider the functional $L$, given by

$$
L=\sum_{i=1}^{a}\left[\int_{0}^{T}\{\dot{g}(t ; i)\}^{2} d t\right]+\sum_{i=1}^{a} \mu(i)\left[\int_{0}^{T}\{g(t ; i)\}^{2} d t\right]
$$

where the $\{\mu(i) ; i=1, \cdots, a\}$ are Lagrange multipliers. The Euler-Lagrange equation [11, p. 53, eq. (2.3.9)] then gives the following necessary condition for optimality:

$$
\mu(i) g^{*}(t ; i)-\ddot{g}^{*}(t ; i)=0
$$

for $i=1, \cdots, a$, where the $g^{*}$ denote the optimum signals and where $\ddot{g}^{*}(t ; i)$ denotes the second time derivative of $g^{*}(t ; i)$.

The optimal signal set $\mathscr{G}^{*}=\left\{g^{*}(t ; i): i=1, \cdots, a\right\}$ thus has to satisfy the following necessary conditions for $i=$ $1, \cdots, a$ :

$$
\begin{aligned}
g^{*}(0 ; i)=g^{*}(T ; i) & =0 \\
\mu(i) g^{*}(t ; i)-\ddot{g}^{*}(t ; i) & =0 \\
\int_{0}^{T}\left\{g^{*}(t ; i)\right\}^{2} d t & =E_{1} .
\end{aligned}
$$

Furthermore, from [11, p. 409 , cond. $N_{1}, S_{1}, S_{2} ;$ p. 373, def. 6.10 ; p. 375 , theorem 6.10$]$, we observe that the necessary conditions (47)-(49) for optimality are also sufficient.

Thus (47)-(49) define solutions to the "relaxed" optimization problem in the absence of the mutually orthogonal simplex constraint. For the fully constrained problem, the optimal signal set $\mathscr{G}^{*}=\left\{g^{*}(t ; i): i=1, \cdots, a\right\}$ must be a simplex set satisfying $(47)-(50)$ and be orthogonal to the optimal signal set $\mathscr{H}^{*}=\{h(t ; l) ; l=1, \cdots, b\}$ for the second sender.

The solutions to the ordinary differential equations (48) with the boundary conditions specified in (47) are sinusoids. We can then specify the optimal signal sets $\mathscr{G}^{*}$ and $\mathscr{H}^{*}$ as follows using the standard procedure for the construction of simplex sets [10, pp. 259-261]. Beginning with sets of orthonormal functions derived from sinusoids, we construct two sets of simplex signals satisfying all the conditions for optimality. Consider the following mutually orthonormal sets of sinusoidal functions defined on the time interval $[0, T]$, assuming without any loss of generality that $a \geq b$ :

$$
\Phi=\left\{\phi(t ; i)=(2 / T)^{1 / 2} \sin n_{i} \pi t / T: i=1, \cdots, a\right\}
$$

and

$$
\Psi=\left\{\psi(t ; l)=(2 / T)^{1 / 2} \sin m_{l} \pi t / T: l=1, \cdots, b\right\}
$$

where $n_{i}$ and $m_{l}$ are positive integers. Note that to obtain mutually orthogonal simplex sets from (50) and (51), we need the sets $\left\{n_{i}: i=1, \cdots, a\right\}$ and $\left\{m_{l}: l=1, \cdots, b\right\}$ to be disjoint. The optimal signal sets $\mathscr{G}^{*}=\left\{g^{*}(t ; i): i=\right.$ $1, \cdots, a\}$ and $\mathscr{H}^{*}=\left\{h^{*}(t ; l): l=1, \cdots, b\right\}$ are then described by

$$
\begin{aligned}
g^{*}(t ; i)= & \left\{a E_{1} /(a-1)\right\}^{1 / 2} \\
& \cdot\left[\{(a-1) / a\} \phi(t ; i)-a^{-1} \sum_{\substack{j=1 \\
j \neq i}}^{a} \phi(t ; j)\right]
\end{aligned}
$$

for $i=1, \cdots, a$, and

$$
\begin{aligned}
h^{*}(t ; l)= & \left\{b E_{2} /(b-1)\right\}^{1 / 2} \\
& \cdot\left[\{(b-1) / b\} \psi(t ; l) \cdots b^{-1} \sum_{\substack{k=1 \\
k \neq l}}^{b} \psi(t ; k)\right]
\end{aligned}
$$

for $l=1, \cdots, b$.

It is easily verified that the signal sets $\mathscr{G}^{*}$ and $\mathscr{H}^{*}$, specified by (52) and (53), respectively, constitute mutually orthogonal simplexes that satisfy conditions (47)-(49). By substituting (52) into (39), we obtain upon simplification that for a given number $a$ of signals for the first sender and given the set $\left\{n_{i}: i=1, \cdots, a\right\}$, the minimum square bandwidth $B_{1}^{2}\left(\mathscr{G}^{*}\right)$ of the optimal signal set $\mathscr{G}^{*}$, which achieves the outer bound in (38), is

$$
B_{1}^{2}\left(\mathscr{G}^{*}\right)=\frac{\pi^{2}}{T^{2}}\left(\frac{1}{a} \sum_{i=1}^{a} n_{i}^{2}\right) .
$$

In an identical manner, it can be shown that the minimum squared bandwidth $B_{2}^{2}\left(\mathscr{H}^{*}\right)$ of the second sender is given by

$$
B_{2}^{2}\left(\mathscr{H}^{*}\right)=\frac{\pi^{2}}{T^{2}}\left(\frac{1}{b} \sum_{i=1}^{b} m_{l}^{2}\right) .
$$

It remains only to pick the sets $\left\{n_{i}: i=1, \cdots, a\right\}$ and $\left\{m_{l}\right.$ : $l=i, \cdots, b\}$ suitably. For the case $a \geq b$, it is easily verified from (54) and (55) that the sum of the squared bandwidths $\left(B_{1}^{2}+B_{2}^{2}\right)$ is minimized by choosing

$$
\left\{m_{l}: l=1, \cdots, b\right\}=\{1, \cdots, b\}
$$

and

$$
\left\{n_{i}: i=1, \cdots, a\right\}=\{b+1, \cdots, b+a\} .
$$

An analogous choice can be made when $b>a$.

We remark here that the orthogonality between the optimal signal sets $\mathscr{G}^{*}$ and $\mathscr{H}^{*}$ in (52), (53), and (56) is achieved by means of frequency-division multiplexing. Thus $\mathscr{G}^{*}$ and $\mathscr{H}^{*}$ can be interpreted as being the signal sets with minimum second-moment bandwidths for which frequency-division multiple access (FDMA) actually achieves the outer bound to the cutoff rate region in (38).

The results obtained for the two-sender single-receiver multiple-access channel can be directly extended to the general multiple-access channel with $M$ senders and one receiver. The optimal signal set for each sender is a simplex set derived from sinusoidal functions and constructed in a manner similar to that for the two-sender multiple- 
access channel. The signal sets for the $M$ senders lie in mutually orthogonal spaces.

It would be interesting to obtain the optimal signal sets for the two senders and the corresponding maximal cutoff rate region when the bandwidths $B_{1}$ and $B_{2}$ are less than those specified by (54) and (55), respectively. This problem remains unsolved to date.

\section{B. Signal Sets with Minimum Out-of-Band Energy}

We define the amount of energy $E\left(\mathscr{G} ; B_{1}\right)$ of the signal set $\mathscr{G}$, lying outside the frequency band $\left[-B_{1}, B_{1}\right]$, i.e., its out-of-band energy, as

$$
E\left(\mathscr{G} ; B_{1}\right)=\sum_{i=1}^{a}\left[\int_{-\infty}^{\infty}|G(f ; i)|^{2} d f-\int_{-B_{1}}^{B_{1}}|G(f ; i)|^{2} d f\right]
$$

or, equivalently, as the $\{\chi(l): l=1, \cdots, b\}$ are Lagrange multipliers. Furthermore, from [11, p. 409, cond. N1, S1, and S2; p. 373, def. 6.10 ; and p. 375 , theorem 6.10$]$, we see that the necessary conditions for optimality are also sufficient.

Thus the solutions to the optimization problem without the mutually orthogonal simplex constraint are defined by (43), (60), (37), and (61). For the fully constrained problem, the optimal signal sets $\mathscr{G}^{*}$ and $\mathscr{H}^{*}$ must additionally constitute simplex sets that are mutually orthogonal.

The optimal modulator designs can then be specified as follows. A comparison of (60) with equations defining prolate spheroidal wave functions $[12$, p. 45] shows that, for $i=1, \cdots, a, g^{*}(t ; i)$ is of the form

$$
g^{*}(t ; i)=k(i) \phi\left[t-\frac{T}{2} ; n(i)\right]
$$

where $k(i)$ is independent of $t, n(i)$ is a nonnegative

$E\left[\mathscr{G} ; B_{1}\right]=\sum_{i=1}^{a}\left[\int_{0}^{T}[g(t ; i)]^{2} d t-\int_{0}^{T} g(t ; i)\left[\int_{0}^{T}\left\{\left(\sin 2 \pi B_{1}(t-\tau)\right) / \pi(t-\tau)\right\} g(\tau ; i) d \tau\right] d t\right]$.

The amount of energy $E\left(\mathscr{H} ; B_{2}\right)$ of the signal set $\mathscr{H}$ lying outside the frequency band $\left[-B_{2}, B_{2}\right]$ can be defined in an identical manner. We wish to identify signal sets with minimal out-of-band energies, as defined by (57), which achieve the outer bounds in (38).

As with the previous optimization problem, we shall proceed to minimize $E\left(\mathscr{G} ; B_{1}\right)$ given by $(57)$, subject to the average energy constraint (43) first, temporarily ignoring the constraint of mutually orthogonal simplex sets. The solutions to this relaxed optimization problem will then be used to obtain solutions to the fully constrained problem.

For the functional $L$, given by

$$
\begin{aligned}
L=\sum_{i=1}^{a}\left[\int_{0}^{T}\{g(t ; i)\}^{2} d t-\int_{0}^{T} g(t ; i)\left\{\int_{0}^{T}\left\{\left(\sin 2 \pi B_{2}(t-\tau)\right) / \pi(t-\tau)\right\} g(\tau ; i) d \tau\right\} d t\right] & \\
& +\sum_{i=1}^{a} \eta(i)\left[\int_{0}^{T}\{g(t ; i)\}^{2} d t\right]
\end{aligned}
$$

where the $\{\eta(i): i=1, \cdots, a\}$ are Lagrange multipliers, the Euler-Lagrange equation $[11$, p. 53, (2.3.9)] gives the following necessary condition for optimality:

$$
\begin{aligned}
& 2\left[g^{*}(t ; i)+\eta(i) g^{*}(t ; i)\right] \\
& \quad=\int_{0}^{T}\left[\left\{\sin 2 \pi B_{1}(t-\tau)\right\} / \pi(t-\tau)\right] g^{*}(\tau ; i) d \tau
\end{aligned}
$$

for $i=1, \cdots, a$, where the $g^{*}$ denote the optimal signals. Upon rewriting (51), we obtain

$$
\begin{aligned}
& g^{*}(t ; i)=\frac{1}{2[1+\eta(i)]} \\
& \cdot \int_{0}^{T}\left[\left\{\sin 2 \pi B_{1}(t-\tau)\right\} / \pi(t-\tau)\right] g^{*}(\tau ; i) d t
\end{aligned}
$$

for $i=1, \cdots, a$. The optimal signal set $\mathscr{G}^{*}=\left\{g^{*}(t ; i)\right.$ : $i=1, \cdots, a\}$ therefore has to satisfy (43) and (60). Similarly, it can be shown that the optimal signals in $\mathscr{H}^{*}=$ $\left\{h^{*}(t ; l): l=1, \cdots, b\right\}$ must satisfy

$$
\begin{aligned}
& h^{*}(t ; l)=\frac{1}{2[1+\chi(l)]} \\
& \cdot \int_{0}^{T}\left[\left\{\sin 2 \pi B_{2}(t-\tau)\right\} / \pi(t-\tau)\right] h^{*}(\tau ; l) d \tau
\end{aligned}
$$

for $l=1, \cdots, b$, in addition to satisfying (37); once again, integer, and $\phi[t ; n(i)]$ is a prolate spheroidal wave function with eigenvalue $\lambda\left[n(i), 2 \pi B_{1} T\right]$ (see Appendix III). (The signals in $\mathscr{G}^{*}$ and $\mathscr{H}^{*}$ are restricted to the time interval $[0, T]$, whereas the functions in [12, p. 45] are restricted to the time interval $[-T / 2, T / 2]$. Hence (62) requires suitably time-shifted versions of the prolate spheroidal wave functions defined in [12].) Similarly, it is seen that $h^{*}(t ; l)$, in (61), is of the form

$$
h^{*}(t ; l)=p(l) \psi\left[t-\frac{T}{2} ; m(l)\right]
$$

for $l=1, \cdots, b$, where $p(l)$ is independent of $t, m(l)$ is a nonnegative integer, and $\psi[t ; m(l)]$ is a prolate spheroidal wave function with eigenvalue $\lambda\left[m(l), 2 \pi B_{2} T\right]$.

In general, the bandwidths $B_{1}$ and $B_{2}$ are not equal and the orthogonality between the sets $\mathscr{G}^{*}$ and $\mathscr{H}^{*}$ is achieved by means of time sharing, that is, by transmitting the signals in $\mathscr{G}^{*}$ and $\mathscr{H}^{*}$ during the nonoverlapping timeintervals $[0, \alpha T]$ and $(\alpha T, T]$, respectively, where $\alpha \in(0,1)$ and is as yet undetermined. When $B_{1}=B_{2}$, an alternative means of achieving orthogonality is frequency-division multiplexing where the signals in $\mathscr{G}^{*}$ and $\mathscr{H}^{*}$ are constructed from a sequence of prolate spheroidal wave func- 
tions with orthogonal frequencies. We shall treat the case where $B_{1}$ is not equal to $B_{2}$ below.

We demonstrate in Appendix III that the optimal signal sets $\mathscr{G}^{*}=\{g(t ; i): i=1, \cdots, a\}$ and $\mathscr{H}^{*} \equiv\left\{h^{*}(t ; l): l=\right.$ $1, \cdots, b\}$, which achieve the outer bounds in (38) with minimum out-of-band energies, are specified by

$$
\begin{array}{r}
g^{*}(t ; i)=\left[a E_{1} /(a-1)\right]^{1 / 2}[\{(a-1) / a\} \phi[t ; n(i)] \\
-a^{-1} \sum_{\substack{j=1 \\
j \neq i}}^{a} \phi[t ; n(j)] \\
h^{*}(t ; l)=\left[b E_{2} /(b-1)\right]^{1 / 2}[\{(b-1) / b\} \psi[t ; m(l)] \\
-b^{-1} \sum_{\substack{k=1 \\
k \neq l}}^{b} \psi[t ; m(k)]
\end{array}
$$

for $l=1, \cdots, b$.

On substituting (64) into (57) and simplifying, we see that the signal set $\mathscr{G}^{*}$, for a given number $a$ of signals, produces a minimal out-of-band energy given by

$$
E\left(\mathscr{G}^{*} ; B_{1}\right)=E_{1}\left[a-\sum_{i=0}^{a-1} \lambda\left[n(i), 2 \pi B_{1} \alpha T\right]\right] \text {. }
$$

In an identical manner, it can be shown for the second sender that the minimal out-of-band energy is

$$
E\left(\mathscr{H}^{*} ; B_{2}\right)=E_{2}\left[b-\sum_{l=0}^{b-1} \lambda\left[m(l), 2 \pi B_{2} \bar{\alpha} T\right]\right]
$$

where $\bar{\alpha}=1-\alpha$.

It still remains to be seen how to pick $\alpha$, and the sets $\{n(i): i=0, \cdots, a-1\}$ and $\{m(l):=0, \cdots, b-1\}$. The signal energy contained in the bandwidth $B_{1}$ of the first sender is

$$
\sum_{i=1}^{a} \int_{-B_{1}}^{B_{1}}\left|G^{*}(f ; i)\right|^{2} d f=E_{1} \sum_{i=0}^{a-1} \lambda\left[n(i), 2 \pi B_{1} \alpha T\right]
$$

We would like to pick $\{n(i): i=0, \cdots, a-1\}$ so as to maximize the right side of (68). In view of (3.1) and (3.6) from Appendix III, we see that this is accomplished by choosing

$$
n(i)=i
$$

for $i=0, \cdots, a-1$. For the signals transmitted by the second sender, the energy contained in the bandwidth $B_{2}$ is, similarly,

$$
E_{2} \sum_{l=0}^{b-1} \lambda\left[m(l), 2 \pi B_{2} \bar{\alpha} T\right]
$$

Again, in view of (3.2) and (3.9) from Appendix III, we see that this in-band energy is greatest when

$$
m(l)=l
$$

for $l=0, \cdots, b-1$.
The best choice of $\alpha \in(0,1)$ is the one that maximizes the "total fractional in-band energy" for the two senders, i.e., the quantity $\left(\sum_{i=0}^{a-1} \lambda\left[n(i), 2 \pi B_{1} \alpha T\right]+\sum_{l=0}^{b-1} \lambda\right.$ $\left.\left[m(l), 2 \pi B_{2} \bar{\alpha} T\right]\right)$. Using (69) and (71), we thus pick $\alpha$ as follows:

$$
\begin{aligned}
\alpha=\arg \max _{\beta \in(0,1)}\left[\begin{array}{r}
a-1 \\
\sum_{i=0} \lambda\left(i, 2 \pi B_{1} \beta T\right)
\end{array}\right. & \\
& \left.+\sum_{l=0}^{b-1} \lambda\left(l, 2 \pi B_{2}(1-\beta) T\right)\right] .
\end{aligned}
$$

The existence of the maximum in (72) is guaranteed by [ 15 , pp. 105, 106].

The orthogonality between the optimal signal sets $\mathscr{G}^{*}$ and $\mathscr{H}^{*}$ in (64), (65), (69), (71), and (72) is achieved by means of time-division multiplexing. This leads to the following interpretation: $\mathscr{G}^{*}$ and $\mathscr{H}^{*}$ are the signal sets with minimum out-of-band energies for which time-division multiple access (TDMA) actually achieves the outer bound to the cutoff rate region in (38).

The extension of the results shown above to the general multiple-access channel with $M$ senders and a single receiver is straightforward. The best choice of signals for each sender is a simplex set derived from prolate spheroidal wave functions with the signal sets for the $M$ senders lying in mutually orthogonal spaces.

\section{Additive Signals, Shot-noise-Limited Multiple-ACCess Optical ChanNel}

The model is shown in Fig. 2. The modulators, channel, and detector constitute the optical portion of the overall optical digital communication system. For senders 1 and 2 , the complex envelopes of the optical fields, which are functions of both time and space, are denoted by $E_{1}(t, \vec{r})$ and $E_{2}(t, \vec{r})$, respectively. The optical fields of the two senders are taken to be additive in the channel. The received signal $N(t)$ represents the counting process associated with the output of an ideal direct-detection device. The counting process is assumed to be an inhomogeneous Poisson process with rate function

$$
\lambda(t)=\frac{\eta}{h \nu} \int_{A}\left|E_{1}(t, \vec{r})+E_{2}(t, \vec{r})\right|^{2} d \vec{r}+\lambda_{0}
$$

where $\eta$ is the quantum efficiency of the detector, $h$ is Planck's constant, $\nu$ is the optical carrier frequency, $A$ is the active surface of the detector, and $\lambda_{0}$ represents the contribution of the dark current in the detector to the total count rate. As indicated in [3], $\lambda_{0}$ also accounts for background radiation when this is characterized by many weak modal components. The assumption that $N(t)$ is a Poisson process is met to a close approximation on the free-space channel for coherent sources. For $i=1,2$, the signal count rates $s_{i}(t)$, which are nonnegative, are related to $E_{i}(t, \vec{r})$ by

$$
s_{i}(t)=\frac{\eta}{h \nu} \int_{A}\left|E_{i}(t, \vec{r})\right|^{2} d \vec{r} .
$$




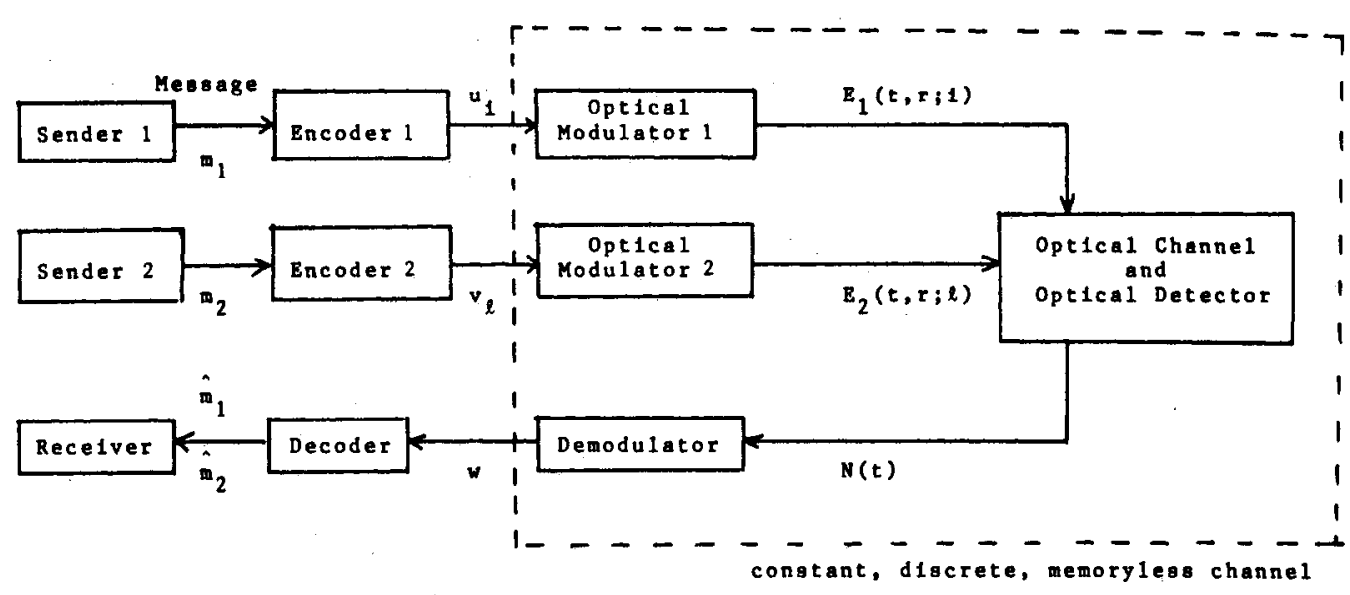

Fig. 2. Model for two-sender one-receiver shot-noise limited optical channel.

The optical modulators for senders 1 and 2 produce signals from the sets $\mathscr{P}_{1}=\left\{E_{1}(t, r ; i): i=1, \cdots, a\right\}$ and $\mathscr{S}_{2}=\left\{E_{2}(t, \vec{r} ; l): \quad l=1, \cdots, b\right\}$, respectively, where $E_{1}(t, \vec{r} ; i)$ is transmitted by sender 1 if his encoder produces the $i$ th codeletter from his alphabet $X_{1}$. All signals are transmitted and received on the time interval $[0, T]$. Associated with the signal sets $\mathscr{S}_{1}$ and $\mathscr{S}_{2}$, respectively, are derived signal sets $\mathscr{D}=\{d(t ; i): i=1, \cdots, a\}$ and $\mathscr{E}=$ $\{e(t ; l): l=1, \cdots, b\}$, where

$$
\begin{aligned}
d(t ; i) & =\left\{\lambda_{1}(t ; i)\right\}^{1 / 2} \\
\lambda_{1}(t ; i) & =\frac{\eta}{h \nu} \int_{A}\left|E_{1}(t, \vec{r} ; i)\right|^{2} d \vec{r}+\lambda_{0} \\
& =s_{1}(t ; i)+\lambda_{0}
\end{aligned}
$$

for $i=1, \cdots, a$, and

$$
\begin{aligned}
e(t ; l) & =\left\{\lambda_{2}(t ; l)\right\}^{1 / 2} \\
\lambda_{2}(t ; l) & =\frac{\eta}{h \nu} \int_{A}\left|E_{2}(t, \vec{r} ; l)\right|^{2} d \vec{r}+\lambda_{0} \\
& =s_{2}(t ; l)+\lambda_{0}
\end{aligned}
$$

for $l=1, \cdots, b$.

The demodulator quantizes the point process observed on $[0, T]$ and produces one of the $c$ symbols from the alphabet $Y$. As seen by the encoder and the decoder, the combination of the optical modulators, optical channel, optical detector, and demodulator forms a discrete channel. The independent increments property of the Poisson process and the constancy of $\lambda_{0}$ make this a constant discrete memoryless channel in the sense of (2). The cutoff rate region of any channel is not reduced by using a finer output quantization; so we examine the limiting case of infinitely fine quantization, that is, when $c=\infty$. When the output quantization is infinitely fine, we demonstrate in Appendix IV that (4)-(7) become

$$
\begin{aligned}
& R_{1 \infty}^{*}(q)=-\ln \sum_{i, j=1}^{a} q_{1}\left(u_{i}\right) q_{1}\left(u_{j}\right) \exp \left(-\alpha_{i j} / 2\right) \\
& R_{2 \infty}^{*}(q)=-\ln \sum_{l, k=1}^{b} q_{2}\left(v_{l}\right) q_{2}\left(v_{k}\right) \exp \left(-\beta_{l k} / 2\right) \\
& R_{3 \infty}^{*}(q)=R_{1 \infty}^{*}(q)+R_{2 \infty}^{*}(q)
\end{aligned}
$$

where $R_{1 \infty}^{*}(q), R_{2 \infty}^{*}(q)$, and $R_{3 \infty}^{*}(q)$ denote, respectively, the values of $R_{1}^{*}(q), R_{2}^{*}(q)$, and $R_{3}^{*}(q)$ for $c=\infty$, and where

$$
\alpha_{i j}=\int_{0}^{T / 2}[d(t ; i)-d(t ; j)]^{2} d t
$$

and

$$
\beta_{l k}=\int_{T / 2}^{T}[e(t ; l)-e(t ; k)]^{2} d t .
$$

We remark here that, as shown in Appendix IV, restricting the signals in $\mathscr{D}$ and $\mathscr{E}$ to lie in the time intervals $[0, T / 2]$ and $[T / 2, T]$, respectively, is a sufficient condition for achieving the upper bounds in (4.10) and (4.11). This restriction imposes a time-division multiple access rule on the transmitters. Then, in a fashion identical to that in which (31) was obtained, we observe that an outer bound on the cutoff rate region of this channel when TDMA is employed is the region of all rate pairs $\left(R_{1}, R_{2}\right)$ satisfying

$$
R_{1} \leq-\ln \left[a^{-1}+\left(1-a^{-1}\right) \exp (-\alpha / 2)\right]
$$

and

$$
R_{2} \leq-\ln \left[b^{-1}+\left(1-b^{-1}\right) \exp (-\beta / 2)\right]
$$

where $\alpha$ and $\beta$ denote the respective maxima of $\alpha_{i j}$ for all $i, j$, and $\beta_{l k}$ for all $l, k$. Moreover, the actual cutoff rate region for TDMA coincides with this outer bound when $\alpha_{i j}=\alpha$ for $i \neq j$, and $\beta_{l k}=\beta$ for $l \neq k$. Also, for each sender, the optimizing $q_{i}$ distribution achieving the bound in (84) is uniform, i.e., $q_{1}\left(u_{i}\right)=a^{-1}$, for $i=1, \cdots, a$, and $q_{2}\left(v_{l}\right)=b^{-1}$, for $l=1, \cdots, b$. Furthermore, since we are restricting the two senders to using TDMA, the region in (84) is only an inner bound on the cutoff rate region of the two sender channel.

The outer bound on the cutoff rate region for TDMA in (84) is maximized when $\alpha$ and $\beta$ are maximal with respect to the functions in $\mathscr{D}=\{d(t ; i): i=1, \cdots, a\}$ and $\mathscr{E}=$ $\{e(t ; l): l=1, \cdots, b\}$, respectively. It remains to identify optimal TDMA signal sets which, in the presence of energy, peak amplitude, and bandwidth constraints to be specified later, achieve the outer bound in (84), with $\alpha$ and $\beta$ taking on the maximum values allowed by the constraints. The choice of the time intervals $[0, T / 2]$ and 
$(T / 2, T]$ for time sharing of the signals is arbitrary. In general, we would like to pick these intervals to be $[0, \theta T]$ and $(\theta T, T]$, respectively, where $\theta \in(0,1)$ is to be chosen suitably. However, as we shall observe later, this makes the problem quite intractable.

Since we have restricted ourselves to TDMA, the multiple-access channel reduces to two independent discrete channels used by the two senders. It therefore suffices to maximize the upper bounds in (84) independently with respect to $\mathscr{D}$ and $\mathscr{E}$, respectively. Thus we shall only present the maximization of the upper bound on $R_{1}$ in (84) subject to energy, peak amplitude, and bandwidth constraints; this is equivalent to maximizing the single-user cutoff rate of sender 1, assuming that all signals are transmitted and received during the time interval $[0, T / 2]$. Subsequently, we shall combine this result with TDMA to provide an obvious inner bound on the cutoff rate region of the two-sender channel with energy, peak amplitude, and bandwidth constraints.

It is also important to point out an essential difference between the optimization procedures used for the Gaussian channel in Section III and those for the present optical channel. Given the alphabet sizes and the energies of the two senders, the achievable outer bound in (38) for the Gaussian channel was not a function of the signal sets $\mathscr{G}$ and $\mathscr{H}$. Thus the necessary and sufficient conditions satisfied by $\mathscr{G}$ and $\mathscr{H}$ to achieve this bound could be used as constraints in the minimization of the bandwidths of these signal sets. The situation for the optical channel is different; as seen from (84), the outer bound on the TDMA cutoff rate region is a function of the signal sets $\mathscr{D}$ and $\mathscr{E}$. We therefore maximize the upper bounds in (84) while restricting the signals in $\mathscr{D}$ and $\mathscr{E}$ to those that achieve these bounds; additional constraints are placed on the energies, peak amplitudes, and bandwidths of these signals.

The cutoff rate for sender 1 is maximized when the achievable upper bound on $R_{1}$ in (84) is greatest; this occurs when $\alpha$ is maximal with respect to the functions in $\mathscr{D}=\{d(t ; i): i=1, \cdots, a\}$. The following four constraints, the first of which is required for the achievability of the upper bound in (84), are imposed on the signals in $\mathscr{D}$.

1) $\alpha_{i j}$, in (82), has the same value whenever $i \neq j$.

2) Average Energy Constraints: We assume that the average energy $\bar{E}_{1}$ of the signals in $\mathscr{I}_{1}$ satisfies

$$
\bar{E}_{1}=a^{-1} \int_{0}^{T / 2} \int_{A} \sum_{i=1}^{a}\left|E_{1}(t, \vec{r} ; i)\right|^{2} d \vec{r} d t \leq \bar{E}_{1, \max }
$$

where $E_{1, \max }$ is the maximum allowable average energy. From (75) and (76), we are led to the following constraints on the average energy $\bar{E}_{d}$ of the signals in $\mathscr{D}$ :

$\bar{E}_{d}=a^{-1} \int_{0}^{T / 2} \sum_{i=1}^{a}\{d(t ; i)\}^{2} d t \leq(\eta / h \nu) \bar{E}_{1, \max }+\lambda_{0} T / 2$.

Now, letting

$$
E_{1}=(\eta / h \nu) \bar{E}_{1, \max }+\lambda_{0} T / 2
$$

we can rewrite (86) as follows:

$$
\int_{0}^{T / 2} \sum_{i=1}^{a}\{d(t ; i)\}^{2} d t \leq a E_{1}
$$

Snyder and Rhodes [3] have shown for the single-user optical channel with infinite bandwidth that there are different categories of optimal design of modulated signals, depending on varying values of the average energy of the signals. The most interesting category is the one in which all the available energy is used up by the modulated signals, that is, the one in which the average energy constraint is satisfied with equality (the region where the "average energy predominates" in [3]). We shall determine the choice of optimal signal set $\mathscr{D}$ which satisfies (88) with equality, that is, which uses up all the available energy.

3) Peak Amplitude Constraints: The amplitudes of the signals in $\mathscr{S}_{1}$ are bounded above by prespecified value $P_{1, \max }$ for all $t \in[0, T / 2]$, and for all locations $\vec{r}$ on the active surface of the detector, as follows:

$$
\left|E_{1}(t, \vec{r} ; i)\right| \leq P_{1, \max }
$$

for $i=1, \cdots, a$. From (75), (76), and (89), we obtain the following constraints on the amplitudes of the signals in $\mathscr{D}$ :

$$
d_{\min } \leq d(t ; i) \leq d_{\max }, \quad t \in[0, T / 2]
$$

for $i=1, \cdots, a$, where $d_{\min }=\lambda_{6}^{1 / 2}$ and

$$
d_{\max }=\left[\frac{\eta A}{h \nu} P_{1, \max }^{2}+\lambda_{0}\right]^{1 / 2} .
$$

4) Bandwidth Constraints: The cutoff rate of sender 1 is maximized subject to the second moment bandwidth constraint. It would be most appropriate to impose this constraint on the signals in the set $\left\{E_{1}(t, \vec{r} ; i): i=1, \cdots, a\right\}$; however, this renders the optimization problem intractable. We therefore constrain the bandwidths of the signals in the set $\mathscr{D}$ instead. If $B_{1}$ denotes the allocated bandwidth, we obtain the following from (12) and (88).

\section{Second Moment Bandwidth Constraints}

Since the signals in $\mathscr{D}$ are at least as large as $d_{\min }$ in amplitude in the time interval $[0, T / 2]$ and vanish outside this interval, conditions for the continuity of the signals at the end points $t=0$ and $t=T / 2$, do not obtain here, unlike in (44). We are thus led to the following constraints:

$$
\int_{0}^{T / 2}\{d(t ; i)\}^{2} d t=E_{1} B_{1}^{2}
$$

for $i=1, \cdots, a$, where $\dot{d}(t ; i)$ is the time derivative of $d(t ; i)$. 
If constraint 1 is satisfied, we obtain on summing both sides of (82) over $i \neq j$ that

$$
\begin{aligned}
\alpha=2[a(a-1)]^{-1} & {\left[(a-1) \int_{0}^{T / 2} \sum_{i=1}^{a}\{d(t ; i)\}^{2} d t\right.} \\
& \left.-\int_{0}^{T / 2} \sum_{\substack{i, j=1 \\
i \neq j}}^{a} d(t ; i) d(t ; j) d t\right] .
\end{aligned}
$$

We now perform the constrained optimization of $\alpha$ with respect to the signals in $\mathscr{D}$. Effecting the following change of variables:

$$
y(t ; i)=d(t ; i)-d_{\min }
$$

for $i=1, \cdots, a$, we obtain from (92) that

$$
\begin{aligned}
\alpha=2[a(a-1)]^{-1} & {\left[(a-1) \int_{0}^{T / 2} \sum_{i-1}^{a}\{y(t ; i)\}^{2} d t\right.} \\
& \left.-\int_{0}^{T / 2} \sum_{\substack{i, j=1 \\
i \neq j}}^{a} y(t ; i) y(t ; j) d t\right] .
\end{aligned}
$$

Thus we can now maximize $\alpha$ with respect to the functions in the set $\mathscr{Y}=\{y(t ; i): i=1, \cdots, a\}$. The following constraints, which follow, respectively, from constraints 1), 2), 3), and 4) on the signals in $\mathscr{D}$, are imposed on the signals in $\mathscr{Y}$.

1a) $\alpha_{i j}$ has the same value whenever $i \neq j$, where

$$
\alpha_{i j}=\int_{0}^{T / 2}[y(t ; i)-y(t ; j)]^{2} d t
$$

2a) Average energy constraint:

$$
\int_{0}^{T / 2} \sum_{i=1}^{a}\left\{y(t ; i)+d_{\min }\right\}^{2} d t=a E_{1} .
$$

3a) Peak-amplitude constraints:

$$
0 \leq y(t ; i) \leq D
$$

for $i=1, \cdots, a$, where

$$
D=d_{\max }-d_{\text {min }}
$$

4a) Second moment bandwidth constraints:

$$
\int_{0}^{T / 2}\{\dot{y}(t ; i)\}^{2} d t=E_{1} B_{1}^{2}
$$

for $i=1, \cdots, a$.

\section{Optimal Signals for the Second Moment}

\section{Bandwidth Constraint}

The optimization problem is one of selecting the signal set $\mathscr{Y}=\{y(t ; i): i=1, \cdots, a\}$ to maximize $\alpha$ in (92), subject to constraints $1 \mathrm{a})-4 \mathrm{a}$ ). Once again, the equidistance constraint 1a) is temporarily ignored in solving this optimi- zation problem. Subsequently, we shall see that among the solutions to the relaxed problem is one that satisfies 1a) which is then the solution to the fully constrained problem.

Define $x_{1}(t)$ and $x_{2}(t ; i)$ for $i=1, \cdots, a, 0 \leq t \leq T / 2$, according to

$$
\begin{aligned}
& \dot{x}_{1}(t)=\sum_{i=1}^{a}\left[\{y(t ; i)\}^{2}+2 d_{\min } y(t ; i)\right] \\
& x_{1}(0)=0
\end{aligned}
$$

and

$$
\begin{aligned}
& \dot{x}_{2}(t ; i)=\{\dot{y}(t ; i)\}^{2} \\
& x_{2}(0 ; i)=0 .
\end{aligned}
$$

Then (96) and (99) become, respectively,

$$
x_{1}(T / 2)=a E_{1}
$$

and

$$
x_{2}(T / 2 ; i)=E_{1} B_{1}^{2}
$$

for $i=1, \cdots, a$. From (100), we obtain

$$
\begin{aligned}
\int_{0}^{T / 2} \sum_{i=1}^{a}\{y(t ; i)\}^{2} d t & =x_{1}(T / 2) \\
& -2 d_{\min } \int_{0}^{T / 2} \sum_{i=1}^{a} y(t ; i) d t
\end{aligned}
$$

A substitution of (104) into (94) yields

$$
\begin{aligned}
& \alpha=2[a(a-1)]^{-1}\left[a(a-1) E_{1}-2 d_{\min }(a-1)\right. \\
& \left.\cdot \int_{0}^{T / 2} \sum_{i=1}^{a} y(t ; i) d t-\int_{0}^{T / 2} \sum_{\substack{i, j=1 \\
i \neq j}}^{a} y(t ; i) y(t ; j) d t\right]
\end{aligned}
$$

Thus, for a fixed value of $E_{1}, \alpha$ is maximized by minimizing

$$
\begin{aligned}
J_{1}=2 d_{\min }(a-1) \int_{0}^{T / 2} & \sum_{i=1}^{a} y(t ; i) d t \\
& +\int_{0}^{T / 2} \sum_{\substack{i, j=1 \\
i \neq j}}^{a} y(t ; i) y(t ; j) d t
\end{aligned}
$$

subject to (100)-(103) and the peak-amplitude constraints (97). Using the Pontryagin minimum principle [13, pp. 306-307], we show in Appendix $V$ that the signal set $\mathscr{D}^{*}=\left\{d^{*}(t ; i): i=1, \cdots, a\right\}$ satisfying the necessary conditions of optimality is the equidistant pulse-position mod- 

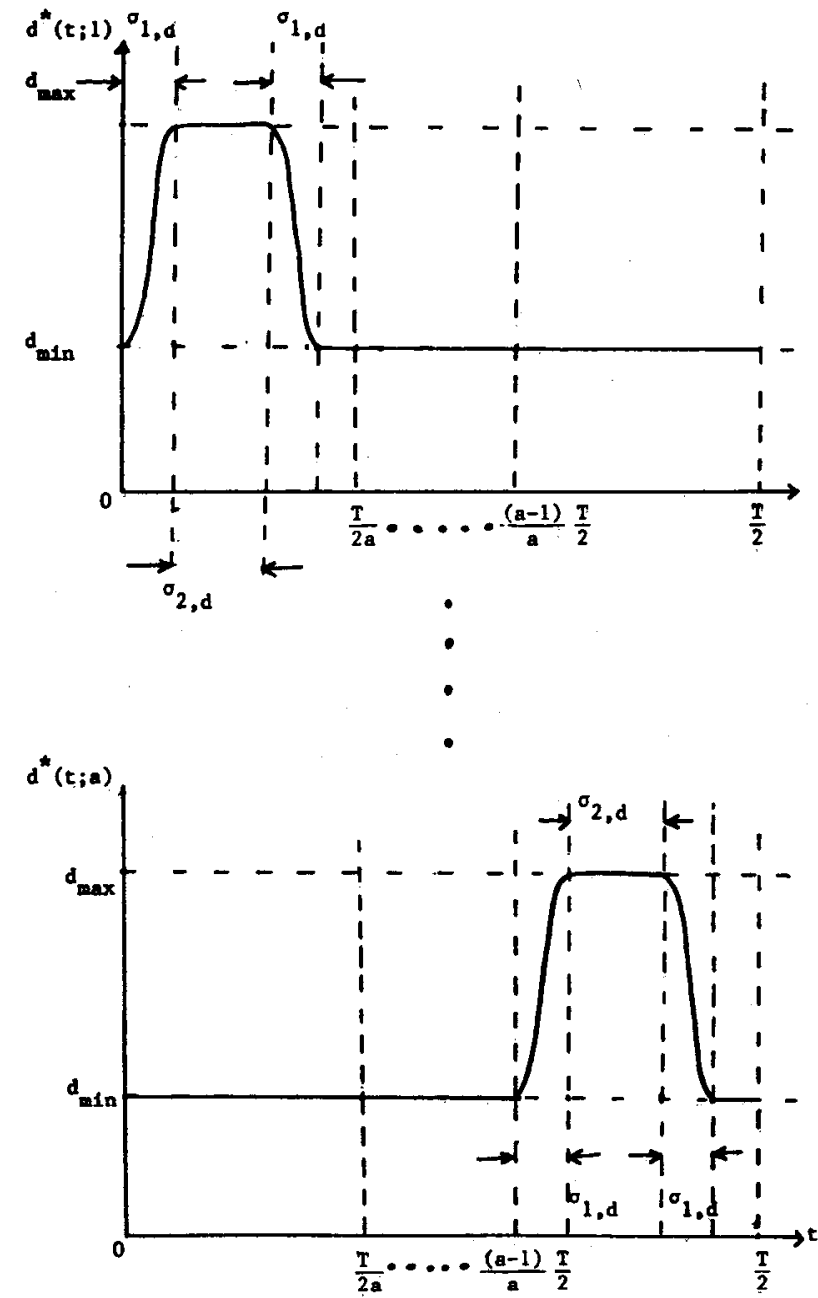

Fig. 3. Optimal PPM signal set $\mathscr{D}^{*}$ for sender 1.
The signal set $\mathscr{D}^{*}$ is optimal for values of the average energy $E_{1}$ in the range $\left[E_{1, \min }, E_{1, \max }\right]$, where $E_{1, \min }$ and $E_{1, \max }$ are given by (5.18) and (5.20), respectively, in Appendix V.

The optimization problem stated in (100)-(106) and (97) is seen to satisfy the conditions of [14, theorem 5, pp. 341], so that the necessary conditions given by the minimum principle are also sufficient. Thus (107)-(110) along with (5.21) $-(5.24)$, indeed, specify the solutions to the optimization problem.

The total "on time" of a pulse in the PPM signal scheme described by (107)-(110) must be less than or equal to $T / 2 a$; thus

$$
2 \sigma_{1, d}+\sigma_{2, d} \leq T / 2 a
$$

or, from $(108)-(110)$,

$$
\frac{\pi^{2}\left(d_{\max }-d_{\min }\right)^{2}}{2 E_{1} B_{1}^{2}}+\frac{\left(E_{1}-E_{1, \min }\right)}{\left(d_{\max }^{2}-d_{\min }^{2}\right)} \leq \frac{T}{2 a},
$$

which imposes a minimum value on the squared bandwidth $B_{1}^{2}$ for the signal set in (107)-(110) to be optimal.

The maximum value of $\alpha$ is obtained from (94) as

$$
\begin{aligned}
\alpha & =(2 / a) \int_{0}^{T / 2} \sum_{i=1}^{a}\left\{y^{*}(t ; i)\right\}^{2} d t \\
& =2 \int_{0}^{T / 2}\left\{y^{*}(t ; i)\right\}^{2} d t
\end{aligned}
$$

since the integral in (112) is the same for $i=1, \cdots, a$. From (93), (107)-(110), and (112) we have

$$
\alpha=2\left[2 \int _ { 0 } ^ { \pi / \omega _ { d } } \left\{\left(\frac{d_{\max }-d_{\min }}{2}\right)\left(1+\cos \left(\omega_{d} t-\pi\right)\right\}^{2} d t\right.\right.
$$

$$
\left.+\left(\dot{d}_{\max }-d_{\min }\right)^{2} \sigma_{2, d}\right]
$$

ulation (PPM) signal set, shown in Fig. 3 and specified by

$$
d^{*}(t ; i)=\left\{\begin{array}{l}
\frac{1}{2}\left[\left(d_{\max }+d_{\min }\right)+\left(d_{\max }-d_{\min }\right) \cos \left\{\omega_{d}\left(t-\frac{\pi}{\omega_{d}}-(i-1) \frac{T}{2 a}\right)\right\}\right], \quad \text { for } \quad(i-1) \frac{T}{2 a} \leq t \leq(i-1) \frac{T}{2 a}+\sigma_{1, d} \\
d_{\max }, \quad(i-1) \frac{T}{2 a}+\sigma_{1, d} \leq t \leq(i-1) \frac{T}{2 a}+\sigma_{1, d}+\sigma_{2, d} \\
\frac{1}{2}\left[\left(d_{\max }+d_{\min }\right)+\left(d_{\max }-d_{\min }\right) \cos \left\{\omega_{d}\left(t-(i-1) \frac{T}{2 a}-\sigma_{1, d}-\sigma_{2, d}\right)\right\}\right], \\
\text { for } \quad(i-1) \frac{T}{2 a}+\sigma_{1, d}+\sigma_{2, d} \leq t \leq(i-1) \frac{T}{2 a}+2 \sigma_{1, d}+\sigma_{2, d} \\
d_{\min }, \quad \text { elsewhere for } 0 \leq t \leq T / 2
\end{array} .\right.
$$

where

$$
\begin{aligned}
\omega_{d} & =\frac{4 E_{1} B_{1}^{2}}{\pi\left(d_{\max }-d_{\min }\right)^{2}} \\
\sigma_{1, d} & =\pi / \omega_{d} \\
\sigma_{2, d} & =\left(E_{1}-E_{1, \min }\right) /\left(d_{\max }^{2}-d_{\min }^{2}\right) .
\end{aligned}
$$

which upon simplification yields

$\alpha=2\left(E_{1}-E_{1, \min }\right)\left(\frac{d_{\text {max }}-d_{\text {min }}}{d_{\text {max }}+d_{\text {min }}}\right)+\frac{3 \pi^{2}}{8 E_{1} B_{1}^{2}}\left(d_{\text {max }}-d_{\text {min }}\right)^{4}$.

On substituting (5.21) into (113) and simplifying, we 
obtain

$$
\begin{aligned}
\alpha= & 2\left(E_{1}-d_{\min }^{2} T / 2\right)\left(\frac{d_{\max }-d_{\min }}{d_{\max }+d_{\min }}\right)-\frac{\pi^{2}\left(d_{\max }-d_{\min }\right)^{3}}{B_{1}^{2}} \\
& \cdot\left[\left\{\left(\frac{d_{\max }+d_{\min }}{2}\right)^{2}+\frac{1}{2}\left(\frac{d_{\max }-d_{\min }}{2}\right)^{2}-d_{\min }^{2}\right)\right\} \\
& \left.\frac{1}{E_{1, \min }}-\frac{3\left(d_{\max }-d_{\min }\right)}{8 E_{1}}\right]
\end{aligned}
$$

Following a procedure identical to that used in obtaining $\mathscr{D}^{*}$, it can be shown that $\beta$ in (84) is maximized by the equidistant PPM signal set $\mathscr{E}^{*}=\left\{e^{*}(t ; l): l=1, \cdots, b\right\}$, defined on the time interval $[T / 2, T]$ and specified by and

$$
\begin{aligned}
E_{2, \max }= & \frac{T}{2 b}\left\{(b-1) d_{\min }^{2}+e_{\max }^{2}\right\} \\
& +\frac{1}{2}\left[\left\{\frac{T}{2 b}\left((b-1) d_{\min }^{2}+e_{\max }^{2}\right)\right\}^{2}\right. \\
& -\frac{2 \pi^{2}\left(e_{\max }-d_{\min }\right)^{2}}{4 B^{2}} \\
& \left.\cdot\left\{e_{\max }^{2}-\left(\frac{e_{\max }+d_{\min }}{2}\right)^{2}-\frac{1}{2}\left(\frac{e_{\max }-d_{\min }}{2}\right)^{2}\right\}\right]^{1 / 2}
\end{aligned}
$$

The signals in (115)-(118) are optimal as long as the squared bandwidth $B_{2}^{2}$ has a minimum value obtained in a manner identical to (111). The maximum value of $\beta$ is

$$
e^{*}(t ; l)=\left\{\begin{array}{c}
\frac{1}{2}\left[\left(e_{\max }+d_{\min }\right)+\left(e_{\max }-d_{\min }\right) \cos \left\{\omega_{e}\left(t-\frac{\pi}{\omega_{e}}-\frac{T}{2}-(l-1) \frac{T}{2 b}\right)\right\}\right], \quad \text { for } \\
\frac{T}{2}+(l-1) \frac{T}{2 b} \leq t \leq \frac{T}{2}+(l-1) \frac{T}{2 b}+\sigma_{1, e} \\
e_{\max }, \quad \frac{T}{2}+(l-1) \frac{T}{2 b}+\sigma_{1, e} \leq t \leq \frac{T}{2}+(l-1) \frac{T}{2 b}+\sigma_{1, e}+\sigma_{2, e} \\
\frac{1}{2}\left[\left(e_{\max }+d_{\min }\right)+\left(e_{\max }-d_{\min }\right) \cos \left\{\omega_{e}\left(t-\frac{T}{2}-(l-1) \frac{T}{2 b}-\sigma_{1, e}-\sigma_{2, e}\right)\right\}\right], \\
\frac{T}{2}+(l-1) \frac{T}{2 b}=\sigma_{1, e}+\sigma_{2, e} \leq t \leq \frac{T}{2}+(l-1) \frac{T}{2 b}+2 \sigma_{1, e}+\sigma_{2, e} \\
d_{\min }, \quad \text { elsewhere for } T / 2 \leq t \leq T
\end{array}\right.
$$

where

$$
\begin{aligned}
\omega_{e} & =\frac{4 E_{2} B_{2}^{2}}{\pi\left(e_{\max }-d_{\min }\right)^{2}}, \\
\sigma_{1, e} & =\pi / \omega_{e} \\
\sigma_{2, e} & =\left(E_{2}-E_{2, \min }\right)\left(e_{\max }^{2}-d_{\min }^{2}\right),
\end{aligned}
$$

and the other undefined quantities are obvious analogs of those used in defining $\mathscr{D}^{*}$.

The set $\mathscr{E}^{*}$ is optimal when the average energy $E_{2}$ of the signals contained in it lies in the range $\left[E_{2, \min }, E_{2, \max }\right]$, where

$$
\begin{gathered}
E_{2, \min }=d_{\min }^{2} \frac{T}{4}+\frac{1}{2}\left[\left(d_{\min }^{2} \frac{T}{2}\right)^{2}+\frac{2 \pi^{2}\left(e_{\max }-d_{\min }\right)^{2}}{B_{2}^{2}}\right. \\
\left.\cdot\left\{\left(\frac{e_{\max }+d_{\min }}{2}\right)^{2}+\frac{1}{2}\left(\frac{e_{\max }-d_{\min }}{2}\right)^{2}-d_{\min }^{2}\right)\right]^{1 / 2}
\end{gathered}
$$

obtained as

$$
\begin{aligned}
\beta= & 2\left(E_{2}-d_{\min }^{2} T / 2\right)\left(\frac{e_{\max }-d_{\min }}{e_{\max }+d_{\min }}\right) \frac{-\pi^{2}\left(e_{\max }-d_{\min }\right)^{3}}{B_{2}^{2}} \\
& \cdot\left[\left\{\left(\frac{e_{\max }+d_{\min }}{2}\right)^{2}+\frac{1}{2}\left(\frac{e_{\max }-d_{\min }}{2}\right)^{2}-d_{\min }^{2}\right)\right\} \\
& \left.\cdot \frac{1}{E_{2, \min }}-\frac{3\left(e_{\max }-d_{\min }\right)}{8 E_{2}}\right] .
\end{aligned}
$$

Combining TDMA with the results above for the two single-user channels, we observe that the maximal achievable outer bound on the cutoff rate region with TDMA, as also an obvious inner bound on the cutoff rate region without any restrictions, is given by (84) with $\alpha$ and $\beta$ being specified by (114) and (121), respectively; the corresponding requirements on minimum bandwidths are given, for example, by (111). For the channel with infinite bandwidth, the modulator signal designs maximizing the cutoff rate region can be obtained by letting $B_{1}$ and $B_{2}$ tend to infinity in (107)-(121). It is easily verified that in this limit, the results in (107)-(121) for each of the two senders 
reduce to those of Snyder and Rhodes [3, pp. 329-331] for the case where the "average energy constraint predominates."

We remark here that, had the intervals used for time sharing by the two senders been chosen to be $[0, \theta T]$ and $(\theta T, T]$, respectively, for some $\theta \in(0,1)$, then in (87), for example, $E_{1}$ becomes a function of $\theta$, i.e., $E_{1}=E_{1}(\theta)$. Consequently, $\alpha$ and $\beta$ in (114) and (121), respectively, become complicated functions of $\theta$. The problem of choosing the "best" values of $\theta$ for $\alpha$ and $\beta$ simultaneously becomes ill-posed as these values may not be the same for $\alpha$ and $\beta$. This additional complexity in the optical channel, which was absent in the Gaussian channel, is caused by the requirement in (90) that the signals in the sets $\mathscr{D}$ and $\mathscr{E}$ be bounded below by $\lambda^{1}{ }^{2}$.

These results can extend to the $M$-sender $(M \geq 3)$ single receiver multiple-access channel in a manner analogous to the case $M=2$.

\section{CONCLUSIONS}

In this paper, we have identified modulated signals which maximize the cutoff rate regions of the additive-signal additive white Gaussian noise and the short-noise limited optical multiple-access channels. Furthermore, when constraints are imposed on signal bandwidth, optimal signal sets for the constrained channel were obtained from among the class of signal sets that are optimal for the unconstrained channel, and the resulting requirements on the bandwidths were determined. The problem of identifying signal sets that yield the maximal cutoff rate region for the band-limited channels, without any a priori restrictions on the feasible sets, remains unsolved to date.

\section{ACKNOWLEDGMENT}

The authors are deeply indebted to Professor James L. Massey for an extremely careful reading of earlier versions of the paper that eliminated a serious flaw, and for myriad constructive suggestions which led to a major revision. The authors also wish to thank Professor Costas Georghiades and the anonymous referees for various helpful comments.

APPENDIX I

\section{DERIVATION OF (15)-(20)}

From (5) and (6) we have

$$
R_{1}^{*}(q)=-\ln \sum_{Y} \sum_{X_{2}} q_{2}(v)\left[\sum_{X_{1}} q_{1}(u) p^{1 / 2}(w / u, v)\right]^{2},
$$

which can be expressed as

$$
\begin{aligned}
R_{1}^{*}(q) & =-\ln \sum_{m=1}^{c} \sum_{l=1}^{b} q_{1}\left(v_{l}\right) \\
& \cdot\left[\sum_{i, j=1}^{a} q_{1}\left(u_{l}\right) q_{1}\left(u_{j}\right)\left\{p\left(w_{m} / u_{i}, v_{l}\right) p\left(w_{m} / u_{j}, v_{l}\right)\right\}^{1 / 2}\right] .
\end{aligned}
$$

On interchanging the order of summation, this becomes

$$
\begin{aligned}
& R_{1}^{*}(q)=-\ln \sum_{i, j=1}^{a} q_{1}\left(u_{i}\right) q_{1}\left(u_{j}\right) \\
& \quad \cdot\left[\sum_{l=1}^{b} \sum_{m=1}^{c} q_{2}\left(v_{l}\right)\left\{p\left(w_{m} / u_{i}, v_{l}\right) p\left(w_{m} / u_{j}, v_{l}\right)\right\}^{1 / 2}\right] .
\end{aligned}
$$

We assume that all the signals in $\mathscr{G}=\{g(t ; i): i=1, \cdots, a\}$ and $\mathscr{H}=\{h(t ; l): l=1, \cdots, b\}$ can be expressed as linear combinations of $N$ orthonormal basis functions in the space of differentiable functions on $[0, T]$ and, therefore, can be represented in signal space by the $N$-dimensional vectors $[\boldsymbol{g}(i)$ : $i=1, \cdots, a]$ and $[h(l): l=1, \cdots, b]$, respectively. Let $\boldsymbol{r}$ and $\boldsymbol{n}$ be $N$-dimensional vectors obtained by projecting $r(t)$ and $n(t)$, respectively, onto the same basis functions. Then from (19),

$$
\boldsymbol{r}=\boldsymbol{g}(i)+\boldsymbol{h}(l)+\boldsymbol{n} .
$$

The $N$ components of $\boldsymbol{n}$ are statistically independent Gaussian random variables, each with mean zero and variance $N_{0} / 2$. From [10, pp. 229-232], we observe that as a consequence of the "theorem of irrelevance," the $N$-dimensional vector $r$ contains all the data relevant to the determination of the transmitted signals using maximum-likelihood decoding.

The inner product on the space of differentiable functions on $[0, T]$ is defined by

$$
(\alpha, \beta)=\int_{0}^{T} \alpha(t) \beta(t) d t,
$$

and the norm of $\alpha$, induced by this inner product, is

$$
\|\alpha\|=\left[\int_{0}^{T}\{\alpha(t)\}^{2} d t\right]^{1 / 2} .
$$

We assume that the demodulator output is unquantized, i.c., that the size $c$ of the demodulator alphabet $Y$ is infinite. Then (1.2) becomes

$$
\begin{aligned}
R_{1 \infty}^{*}(q)=-\ln & \sum_{i, j=1}^{a} q_{1}\left(u_{i}\right) q_{1}\left(u_{j}\right) \\
: & {\left[\sum_{l=1}^{b} q_{2}\left(v_{l}\right) \int_{-\infty}^{\infty}\left\{F_{i l}(\gamma) F_{j l}(\gamma)\right\}^{1 / 2} d \gamma\right] }
\end{aligned}
$$

where $R_{1 \infty}^{*}(q)$ denotes the value of $R_{1}^{*}(q)$ with $c=\infty$, and $F_{i l}(\cdot)$ is the probability density function of the vector $r$, given that the vectors $\boldsymbol{g}(i)$ and $\boldsymbol{h}(l)$ are transmitted over the channel.

Now, $F_{i l}(\gamma)$ is given by

$$
F_{i /}(\gamma)=\left(\pi N_{0}\right)^{-1 / 2} \exp \left\{-\|\boldsymbol{\gamma}-\boldsymbol{g}(i)-\boldsymbol{h}(l)\|^{2} / N_{0}\right\}
$$

and thus

$$
\begin{aligned}
\int_{-\infty}^{\infty} & \left\{F_{l l}(\gamma) F_{j l}(\gamma)\right\}^{1 / 2} \boldsymbol{d} \gamma \\
& =\exp \left[-\|(\boldsymbol{g}(i)+\boldsymbol{h}(l)\}-\{\boldsymbol{g}(j)+\boldsymbol{h}(l)\}\|^{2} / 4 N_{0}\right] \\
& =\exp \left\{-\|\boldsymbol{g}(i)-\boldsymbol{g}(j)\|^{2} / 4 N_{0}\right\} \\
& =\exp \left\{-\xi_{i j} / 4 N_{0}\right\}
\end{aligned}
$$

where

$$
\begin{aligned}
\xi_{i j} & =\|\boldsymbol{g}(i)-\boldsymbol{g}(j)\|^{2} \\
& =\int_{0}^{T}[g(t ; i)-g(t ; j)]^{2} d t,
\end{aligned}
$$


from (1.5). Thus, from (1.6),

$$
R_{1 \infty}^{*}(q)=-\ln \sum_{i, j=1}^{a} q_{1}\left(u_{i}\right) q_{1}\left(u_{j}\right) \exp \left(-\xi_{i j} / 2 N_{0}\right) \text {. }
$$

Equations (16) and (17) along with (19) and (20) can be derived in an identical manner.

\section{APPENDIX II}

\section{PRoOF OF ORThOgONALITY BeTWEeN $\mathscr{G}$ AND $\mathscr{H}$ IN SECTION III}

We show that for

$\gamma_{i j l k}=\int_{0}^{T}[\{g(t ; i)-g(t ; j)\}+\{h(t ; l)-h(t ; k)\}]^{2} d t$

to have the same value whenever $i \neq j$ and $l \neq k$, given that $\xi_{i j}$ from (18) and $\eta_{l k}$ from (19) are invariant, it is both necessary and sufficient that the sets $\mathscr{G}=\{g(t ; i): i=1, \cdots, a\}$ and $\mathscr{H}=$ $\{h(t ; l): l=1, \cdots, b\}$ lie in mutually orthogonal vector spaces. From (2.1), we may write

$$
\begin{aligned}
\gamma_{i j l k}= & \int_{0}^{T}\{g(t ; i)-g(t ; j)\}^{2} d t \\
& +\int_{0}^{T}\{h(t ; l)-h(t ; k)\}^{2} d t \\
& +2 \int_{0}^{T}\{g(t ; i)-g(t ; j)\}\{h(t ; l)-h(t ; k)\} d t .
\end{aligned}
$$

The first two integrals in (2.2) are constant for all $i \neq j$ and $l \neq k$. Thus the condition for $\gamma_{i j l k}$ to be constant is that

$$
\int_{0}^{T}\{g(t ; i)-g(t ; j)\}\{h(t ; l)-h(t ; k)\} d t=K
$$

for all $i \neq j$ and $l \neq k$, where $K$ is a constant. Thus (2.3) must hold on interchanging $i$ and $j$; that is, it must hold that

$$
\int_{0}^{T}\{g(t ; j)-g(t ; i)\}\{h(t ; l)-h(t ; k)\} d t=K .
$$

However, from (2.3), we see that the integral in (2.4) is equal to $-K$. This is satisfied if and only if $K=0$, that is, if and only if the two signal sets $\mathscr{G}=\{g(t ; i): i=1, \cdots, a\}$ and $\mathscr{H}=\{h(t ; l)$ : $l=1, \cdots, b\}$ lie in mutually orthogonal spaces.

\section{APPENDIX III}

DeRIVATION OF (64)

Let $\{n(i): i=0, \cdots, a-1\}$ and $\{m(l): l=0, \cdots, b-1\}$ be subsets of the set of natural numbers $\{0,1,2, \cdots\}$. We assume that

$$
n(i) \leq n(i+1)
$$

for $i=0, \cdots, a-1$, and

$$
m(l) \leq m(l+1)
$$

for $l=0, \cdots, b-1$.

Consider the sets $C$ and $E$ of orthogonal functions defined on the time intervals $[0, \alpha T]$ and $(\alpha T, T]$, respectively, by

$$
C=\{c[t ; n(i)] ; i=0, \cdots, a-1\}
$$

and

$$
E=\{e[t ; m(l)]:=0, \cdots, b-1\}
$$

For $i$ and $j$ in $\{0, \cdots, a-1\}$, the functions in the set $C$ satisfy

$$
\begin{aligned}
\int_{0}^{\alpha T} c[t ; n(i)] c[t ; n(j)] d t & =0, \quad i \neq j \\
& =\lambda\left[n(i), 2 \pi B_{1} \alpha T\right], \quad i=j,
\end{aligned}
$$

$$
\lambda\left[n(i), 2 \pi B_{1} \alpha T\right]>\lambda\left[n(i+1), 2 \pi B_{1} \alpha T\right],
$$

and

$$
\begin{aligned}
& \lambda\left[n(i), 2 \pi B_{1} \alpha T\right] c[t ; n(i)] \\
& \quad=\int_{0}^{\alpha T}\left[\left\{\sin 2 \pi B_{1}(t-s)\right\} / \pi(t-s)\right] c[s ; n(i)] d s .
\end{aligned}
$$

In a similar manner, for $l, k=0, \cdots, b-1$, the functions in $E$ satisfy

$$
\begin{aligned}
\int_{\alpha T}^{T} e[t ; m(l)] e[t ; m(k)] d t & =0, \quad l \neq k \\
& =\lambda\left[m(l), 2 \pi B_{2} \bar{\alpha} T\right], \quad l=k,
\end{aligned}
$$

$$
\lambda\left[m(l), 2 \pi B_{2} \bar{\alpha} T\right]>\lambda\left[m(l+1), 2 \pi B_{2} \bar{\alpha} T\right]
$$

where $\bar{\alpha}=1-\alpha$, and

$\lambda\left[m(l), 2 \pi B_{2} \bar{\alpha} T\right] e[t ; m(l)]$

$$
=\int_{T / 2}^{T}\left[\left\{\sin 2 \pi B_{2}(t-s) / \pi(t-s)\right] e[s ; m(l)] d s .\right.
$$

The sets $\Phi$ and $\Psi$, where

$\Phi=\{\phi[t ; n(i)]=c[t ; n(i-1)] /$

$$
\left.\left\{\lambda\left[n(i-1), 2 \pi B_{1} \alpha T\right]\right\}^{1 / 2}: i=1, \cdots, a\right\}
$$

and

$\Psi=\left\{\psi[t ; m(l)]=e\left[t ; m(l-1), 2 \pi B_{2} \bar{\alpha} T\right] /\right.$

$$
\left\{\lambda\left[m(l-1), 2 \pi B_{2} \bar{\alpha} T\right]^{1 / 2}: l=1, \cdots, b\right\}
$$

are, therefore, sets of orthonormal functions on $[0, \alpha T]$ and $(\alpha T, T]$, respectively. These are the prolate spheroidal wave functions.

The simplex set $\mathscr{G}^{*}=\left\{g^{*}(t ; i): i=1, \cdots, a\right\}$, constructed from (3.11) and satisfying (43) and (60), is seen to be specified by

$$
\begin{aligned}
g^{*}(t ; i) & =\left[a E_{1} /(a-1)\right]^{1 / 2} \\
\cdot & {\left[\{(a-1) / a\} \phi[t ; n(i)]-a^{-1} \sum_{\substack{j=1 \\
j \neq i}}^{a} \phi[t ; n(j)]\right] }
\end{aligned}
$$

for $i=1, \cdots, a$ and $0 \leq t \leq a T$.

Similarly, using (3.12) we can construct a simplex set $\mathscr{H}^{*}=$ $\left\{h^{*}(t ; l): l=1, \cdots, b\right\}$ such that (37) and (61) are satisfied. This set is specified by

$$
\begin{aligned}
h^{*}(t ; l)=\left[b E_{2} /(b-1)\right]^{1 / 2} & {\left[\{(b-1) / b\} \psi[t ; m(l)]-b^{-1} \sum_{\substack{k=1 \\
k \neq l}}^{b} \psi[t ; m(k)]\right] }
\end{aligned}
$$




\section{APPENDIX IV}

\section{DERIVATION OF (79)-(81)}

When sender 1 and sender 2 transmit the $i$ th and $j$ th codeletters from their code alphabets $X_{1}$ and $X_{2}$, respectively, we see from (73) that the rate $\lambda_{i l}(t)$ of the counting process associated with the output of the optical detector is

$$
\lambda_{i l}(t)=\frac{\eta}{h \nu} \int_{A}\left|E_{1}(t, \vec{r} ; i)+E_{2}(t, \vec{r} ; l)\right|^{2} d r+\lambda_{0}
$$

for $i=1, \cdots, a$. Upon expanding the right side of (4.1) and using (74), we obtain

$$
\begin{aligned}
\lambda_{i l}(t)=s_{1}(t ; i) & +s_{2}(t ; l) \\
& +\frac{2 \eta}{h \nu} \int_{A} \operatorname{Re}\left[E_{1}(t, \vec{r} ; i) E_{2}^{*}(t, \vec{r} ; l)\right] d \vec{r}+\lambda_{0}
\end{aligned}
$$

where $\operatorname{Re}[\cdot]$ and $[\cdot]^{*}$ denote, respectively, the real part and the complex conjugate of $[\cdot]$.

For a Poisson process with rate $\lambda(t)$, the probability of observing $n$ points during $[0, T]$ in $n$ disjoint intervals $\left[t_{1}, t_{1}+\Delta t_{1}\right)$, $\left[t_{2}, t_{2}+\Delta t_{2}\right), \cdots,\left[t_{n}, t_{n}+\Delta t n\right)$ is approximated to $o\left(\max _{i} \Delta t_{i}\right)$ by

$$
\left(\prod_{l=1}^{n} \lambda\left(t_{l}\right)\right) \exp \left(-\int_{0}^{T} \lambda(t) d t\right) \Delta t_{1} \Delta t_{2} \cdots \Delta t_{n}
$$

for $n \geq 1$, and by

$$
\exp \left(-\int_{0}^{T} \lambda(t) d t\right)
$$

for $n=0$.

When the quantization of the output is infinitely fine, that is, when $c=\infty, R_{1 \infty}^{*}(q)$ can be written from (1.2) in Appendix I as

$$
R_{1 \infty}(q)=-\ln \sum_{i, j=1}^{a} q_{1}\left(u_{i}\right) q_{1}\left(u_{j}\right)\left[\sum_{l=1}^{b} q_{2}\left(v_{l}\right) f(i, j, l)\right]
$$

where

$$
f(i, j, l)=\sum_{m=1}^{\infty}\left\{p\left(w_{m} / u_{i}, v_{l}\right) p\left(w_{m} / u_{j}, v_{l}\right)\right\}^{1 / 2}
$$

From (4.2)-(4.4) and (4.6), we have

$$
\begin{aligned}
f(i, j, l) & =\exp \left[-1 / 2 \int_{0}^{T}\left\{\lambda_{i l}(t)+\lambda_{j l}(t)\right\} d t\right] \\
\cdot & {\left[1+\sum_{n=1}^{\infty} \iint \cdots \int \prod_{l=1}^{n}\left\{\lambda_{i l}(t) \lambda_{j l}(t)\right\}^{1 / 2} d t_{1} d t_{2} \cdots d t_{n}\right] }
\end{aligned}
$$

where the integration is over the region $0 \leq t_{1} \leq t_{2} \leq \cdots \leq t_{n} \leq T$. By extending this range of integration to $0 \leq t_{i} \leq T$ for $i=$ $1, \cdots, n$, and dividing by $n$ ! to compensate for this extension, we obtain

$$
f(i, j, l)=\exp \left[-\frac{1}{2} \int_{0}^{T}\left\{\left(\lambda_{i l}(t)\right)^{1 / 2}-\left(\lambda_{j l}(t)\right)^{1 / 2}\right\}^{2} d t\right] .
$$

Defining $f^{\prime}(i, j, l)$ by

$$
f^{\prime}(i, j, l)=\ln f(i, j, l),
$$

and using Jensen's inequality in (4.5), we obtain

$$
\begin{array}{r}
R_{1 \infty}^{*}(q) \leq-\ln \left[\sum_{i, j=1}^{a} q_{1}\left(u_{i}\right) q_{1}\left(u_{j}\right)\right. \\
\left.\cdot \exp \left\{\sum_{l=1}^{b} q_{2}\left(v_{l}\right) f^{\prime}(i, j, l)\right\}\right],
\end{array}
$$

with equality holding in (4.10) if and only if $f^{\prime}(i, j, l)$ is the same for $l=1, \cdots, b$. In an identical manner, it can be shown that

$$
\begin{array}{r}
R_{2 \infty}^{*}(q) \leq-\ln \left[\sum_{l, k=1}^{b} q_{2}\left(v_{l}\right) q_{2}\left(v_{k}\right)\right. \\
\left.\cdot \exp \left\{\sum_{i=1}^{a} q_{1}\left(u_{i}\right) g^{\prime}(i, l, k)\right\}\right]
\end{array}
$$

where

$$
g^{\prime}(i, l, k)=-\frac{1}{2} \int_{0}^{T}\left\{\left(\lambda_{i l}(t)\right)^{1 / 2}-\left(\lambda_{i k}(t)\right)^{1 / 2}\right\}^{2} d t
$$

with equality holding in (4.12) if and only if $g^{\prime}(i, l, k)$ is the same for $i=1, \cdots, a$.

By expanding (4.9) and (4.12), it is easily verified that a sufficient condition for equality to hold in (4.10) and (4.11) is that for each $t \in[0, T]$ and $\vec{r} \in A$,

$$
E_{1}(t, \vec{r} ; i) E_{2}(t, \vec{r} ; l)=0
$$

for $i=1, \cdots, a$ and $l=1, \cdots, b$. An implication of (4.13) is that, for each $t \in[0, T]$,

$$
s_{1}(t ; i) s_{2}(t ; l)=0,
$$

for $i=1, \cdots, a$ and $l=1, \cdots, b$. This can be achieved by means of time sharing between the signals in $\mathscr{S}_{1}$ and $\mathscr{S}_{2}$. Thus we have

$$
s_{1}(t ; i)=0, \quad \frac{T}{2} \leq t \leq T
$$

for $i=1, \cdots, a$, and

$$
s_{2}(t ; l)=0, \quad 0 \leq t \leq \frac{T}{2}
$$

for $l=1, \cdots, b$. Then using (73), (75), (76), (78), (4.15), and (4.16) in (4.8), we obtain

$$
\begin{aligned}
f(i, j)= & \exp \left[-\frac{1}{2} \int_{0}^{T / 2}\left\{\left(\lambda_{1}(t ; i)\right)^{1 / 2}-\left(\lambda_{1}(t ; j)\right)^{1 / 2}\right\}^{2} d t\right. \\
& \left.-\frac{1}{2} \int_{T / 2}^{T}\left\{\left(\lambda_{2}(t ; l)\right)^{1 / 2}-\left(\lambda_{2}(t ; l)\right)^{1 / 2}\right\}^{2} d t\right] \\
= & \exp \left[-\frac{1}{2} \int_{0}^{T / 2}\{d(t ; i)-d(t ; j)\}^{2} d t\right]
\end{aligned}
$$

Defining $\alpha_{i j}$ by

$$
\alpha_{i j}=\int_{0}^{T / 2}\{d(t ; i)-d(t ; j)\}^{2} d t,
$$

it is seen from (4.10), (4.17), and (4.18) that

$$
R_{1 \infty}^{*}(q)=-\ln \left[\sum_{i, j=1}^{a} q_{1}\left(u_{i}\right) q_{2}\left(u_{j}\right) \exp \left(-\alpha_{i j} / 2\right)\right]
$$

which is (79).

In an identical fashion, by defining $\beta_{l k}$ as

$$
\beta_{l k}=\int_{T / 2}^{T}\{e(t ; l)-e(t ; k)\}^{2} d t
$$


it can be shown that

$$
R_{2 \infty}^{*}(q)=-\ln \left[\sum_{l, k=1}^{b} q_{2}\left(v_{l}\right) q_{2}\left(v_{k}\right) \exp \left(-\beta_{l k} / 2\right)\right] \text {, }
$$

which is (80).

Finally, for $c=\infty,(7)$ can be written as

$$
\begin{array}{r}
R_{3 \infty}^{*}(q)=-\ln \left[\sum_{i, j=1}^{a} \sum_{l, k=1}^{b} q_{1}\left(u_{i}\right) q_{1}\left(u_{j}\right) q_{2}\left(v_{l}\right) q_{2}\left(v_{k}\right)\right. \\
\cdot \exp \left\{-\frac{1}{2} \int_{0}^{T}\left\{\left(\lambda_{i l}(t)\right)^{1 / 2}-\left(\lambda_{j k}(t)\right)^{1 / 2}\right\}^{2} d t\right] .
\end{array}
$$

Using (73), (75) -(78), (4.15), and (4.16) in (4.21), we obtain upon simplication that

$$
\begin{aligned}
R_{3 \infty}^{*}(q)= & -\ln \left[\sum_{i, j=1}^{a} q_{1}\left(u_{i}\right) q_{1}\left(u_{j}\right)\right. \\
& \cdot \exp \left\{-\frac{1}{2} \int_{0}^{T / 2}\{d(t ; i)-d(t ; j)\}^{2} d t\right\} \\
& \cdot \sum_{l, k=1}^{b} q_{2}\left(v_{l}\right) q_{2}\left(v_{k}\right) \\
& \left.\cdot \exp \left\{-\frac{1}{2} \int_{T / 2}^{T}\{e(t ; l)-e(t ; k)\}^{2} d t\right\}\right] .
\end{aligned}
$$

Using (4.18)-(4.20) in (4.22), we obtain

$$
R_{3 \infty}^{*}(q)=R_{1 \infty}^{*}(q)+R_{2 \infty}^{*}(q) \text {, }
$$

which is (81).

\section{APPENDIX V}

DeRIVATION OF (107)-(110)

The Hamilton $H$ is given by

$$
\begin{aligned}
& H\left[x_{1}(t), x_{2}(t), p_{1}(t), p_{2}(t), y(t), \dot{y}(t)\right] \\
& =2 d_{\min }(a-1) \sum_{i=1}^{a} y(t ; i)+\sum_{\substack{i, j=1 \\
i \neq j}}^{a} y(t ; i) y(t ; j) \\
& \quad+p_{1}(t) \sum_{i=1}^{a}\left[\{y(t ; i)\}^{2}+2 d_{\min } y(t ; i)\right] \\
& \quad+\sum_{i=1}^{a} p_{2}(t ; i)\{\dot{y}(t ; i)\}^{2} .
\end{aligned}
$$

With $(\cdot)$ and $[\cdot]^{T}$ denoting $d(\cdot) / d t$ and the transpose of $[\cdot]$, respectively, the following necessary conditions for $y^{*}(t)=$ $\left[y^{*}(t ; 1), y^{*}(t ; 2), \cdots, y^{*}(t ; a)\right]^{T}$ and $\dot{y}^{*}(t)=\left[\dot{y}^{*}(t ; 1)\right.$, $\left.\dot{y}^{*}(t ; 2), \cdots, \dot{y}^{*}(t ; a)\right]^{T}$ to solve the problem defined by $(106)$, (100)-(103), and (97) are provided, respectively, by the Pontryagin minimum principle $[13$, pp. $306-307$, Table $5-1$, line 4 columns 1-4]:

$$
\begin{aligned}
\dot{p}_{1}^{*}(t) & =0, \text { that is } p_{1}^{*}(t) \equiv p_{1}^{*}(T / 2)=p_{1}, \text { a constant, } \\
\dot{p}_{2}^{*}(t ; i) & =0, \text { that is } p_{2}^{*}(t ; i)=p_{2}^{*}(T / 2 ; i)=p_{2}(i),
\end{aligned}
$$

a constant,

for $i=1, \cdots, a$,

$H\left[x_{1}^{*}, \boldsymbol{x}_{2}^{*}, p_{1}, \boldsymbol{p}_{2}^{*}, \boldsymbol{y}^{*}, \dot{y}^{*}\right] \leq H\left[x_{1}^{*}, \boldsymbol{x}_{2}^{*}, p_{1}, \boldsymbol{p}_{2}, \boldsymbol{y}, \dot{\boldsymbol{y}}\right]$, and

$$
\begin{aligned}
H\left[x_{1}^{*}(t), \boldsymbol{x}_{2}^{*}(t), p_{1}, \boldsymbol{p}_{2}, \boldsymbol{y}^{*}(t), \dot{y}^{*}(t)\right] \\
=H\left[x_{1}^{*}(T / 2), \boldsymbol{x}_{2}^{*}(T / 2), p_{1}, p_{2}, y^{*}(T / 2), \dot{y}^{*}(T / 2)\right],
\end{aligned}
$$

Furthermore, no conditions are imposed on $p_{1}$, and $p_{2}=$ $\left[p_{2}(1), p_{2}(2), \cdots, p_{2}(a)\right]^{T}$.

To satisfy (5.5), by setting $d H / d t=0$, wc obtain that, for all $t \in[0, T / 2]$, the following equation is satisfied:

$$
\begin{array}{r}
\sum_{i=1}^{a} \dot{y}^{*}(t ; i)\left[p_{2}(i) \ddot{y}^{*}(t ; i)+p_{1} y^{*}(t ; i)+\sum_{\substack{j=1 \\
j \neq i}}^{a} y^{*}(t ; i)\right. \\
\left.+d_{\min }\left(p_{1}+a-1\right)\right]=0
\end{array}
$$

where $\ddot{y}(t ; i)$ denotes the second time derivative of $y(t ; i)$.

Now, in (106), we observe that both integrands in $J_{1}$ are nonnegative. The smallest possible value of $J_{1}$ is thus zero and occurs when each integrand is zero almost everywhere (a.e.) in $[0, T / 2]$. In view of constraint (97), it is seen that the second integrand in (106) is zero a.e. if and only if a.e. in [0,T/2] either all the signals in $\mathscr{Y}$, or all but one, take on the value zero. The fịst integrand is zero a.e. if and only if a.e. in $[0, T / 2]$ all the signals in $\mathscr{Y}$ have value zero; in this event, however, the terminal constraint (102) is violated. Hence $J_{1}$ in (106) is minimized when a.e. in $[0, T / 2]$ either all, or all but one of the signals in the optimal signal set $\mathscr{Y}^{*}$ take on a zero value.

We can satisfy (5.6) by setting

$$
\begin{aligned}
& \dot{y}^{*}(t ; i)\left[p_{2}(i) \ddot{y}^{*}(t ; i)+p_{1} y^{*}(t ; i)+\sum_{\substack{j=1 \\
j \neq i}}^{a} y^{*}(t ; i)\right. \\
& \left.+d_{\min }\left(p_{1}+a-1\right)\right]=0
\end{aligned}
$$

for each $t \in[0, T / 2]$ and for $i=1, \cdots, a$. This is satisfied for each $t \in[0, T / 2]$ and for a particular $i$ if either

$$
\dot{y}^{*}(t ; i)=0
$$

or

$$
\begin{aligned}
p_{2}(i) \ddot{y}^{*}(t ; i)+p_{1} y^{*}(t ; i)+ & \sum_{\substack{j=1 \\
j \neq 1}}^{a} y^{*}(t ; i) \\
& +d_{\min }\left(p_{1}+a-1\right)=0 .
\end{aligned}
$$

If $y^{*}(t ; i)$ is nonzero for a fixed $t \in[T / 2]$, then in view of the previous discussion, (5.9) simplifies to

$$
p_{2}(i) \ddot{y}^{*}(t ; i)+p_{1} y^{*}(t ; i)+d_{\min }\left(p_{1}+a-1\right)=0 .
$$

The solution to $(5.10)$ is a raised cosine, that is, $y^{*}(t ; i)$ is of the form

$$
y^{*}(t ; i)=A_{i}\left[1+\cos \omega_{i}\left(t-\tau_{i}\right)\right]
$$

where, in accordance with (97), $0<A_{i} \leq D / 2$, and where $\omega_{i}$ and $\tau_{i}$ are yet to be determined. From (5.10) and (5.11), we obtain

$$
p_{1}=-d_{\min }(a-1) /\left(A_{i}+d_{\text {min }}\right)
$$


and

$$
p_{2}(i)=p_{1} / \omega_{i}^{2} \text {. }
$$

From (5.2), $p_{1}$ is a constant; thus from (5.12), $A_{i}$ is the same for all $i$, that is,

$$
A_{i} \equiv A, \quad \text { for } i=1, \cdots, a .
$$

It then follows from (5.11) and (5.12), respectively, that

$$
y^{*}(t ; i)=A\left[1+\cos \omega_{i}\left(t-\tau_{i}\right)\right]
$$

and

$$
p_{1}=-d_{\min }(a-1) /\left(A+d_{\min }\right) .
$$

Thus far, we have seen that for each $t \in[0, T / 2]$, either all or all but one of the signals in the optimal set $\mathscr{Y}^{*}$ take on the value zero, simultaneously satisfying (5.8). Furthermore, the signal with the nonzero value, say $y^{*}(t ; i)$, satisfies either (5.8) or (5.14).

With all the signals in $\mathscr{Y}^{*}$ being zero at some $t \in[0, T / 2]$, and satisfying (5.8), the Hamiltonian $H$ in (5.1) has the value zero. When the signal $y^{*}(t ; i)$ alone satisfies (5.14), it is verified from (5.1) and (5.12)-(5.15) that $H=0$, in accordance with (5.5). When $y^{*}(t ; i)$ alone is nonzero and satisfies $(5.8)$, we see that (5.5) is satisfied if

$$
\begin{aligned}
& 2 d_{\min }(a-1) y^{*}(t ; i)-\left[d_{\min }(a-1) /\left(A_{i}+d_{\min }\right)\right] \\
& \cdot\left[\left\{y^{*}(t ; i)\right\}^{2}+2 d_{\min } y^{*}(t ; i)\right]=0,
\end{aligned}
$$

which upon simplification yields

$$
y^{*}(t ; i)=2 A \text {. }
$$

Thus, at any $t \in[0, T / 2]$, either all or all but one of the signals in $\mathscr{Y}^{*}$ have a zero value. The nonzero signal is either equal to $2 A$, as in (5.16), or is given by (5.14), where $\omega_{i}$ will be shown to be chosen so as to satisfy (103). For this choice of signals, we observe in (94) that the second integral vanishes whereas the first one increases with $A$. Thus $\alpha$ in (94) is maximized when $A$ takes on its greatest value, that is, when $A=D / 2$ (from (5.11)).

Expressing the signals in $\mathscr{Y}^{*}$ in terms of the signals in $\mathscr{D}^{*}=$ $\{d(t ; i): i=1, \cdots, a\}$ by the use of (94), we thus conclude that $\alpha$ in (92) is maximized by the equidistant pulse-position modulation (PPM) signal set given by (107)-(110).

In (110), $E_{1, \min }$ is the smallest value of the average energy $E_{1}$ for which $\mathscr{D}^{*}$ is optimal and corresponds to $\sigma_{2, d}=0 . E_{1, \min }$ is obtaincd from

$$
E_{1, \min }=\int_{0}^{T / 2}\left\{d^{*}(t ; i)\right\}^{2} d t
$$

which, from (108) $-(110)$, with $\sigma_{2 d}=0$ and $E_{1}=E_{\min }$, simplifies to

$$
\begin{aligned}
E_{1, \min }= & d_{\min }^{2} \frac{T}{2}+\frac{\pi^{2}\left(d_{\max }-d_{\min }\right)^{2}}{2 B_{1}^{2} E_{1, \min }} \\
& \cdot\left[\left(\frac{d_{\mathrm{max}}+d_{\mathrm{min}}}{2}\right)^{2}+\frac{1}{2}\left(\frac{d_{\max }-d_{\min }}{2}\right)^{2}-d_{\min }^{2}\right] .
\end{aligned}
$$

Upon solving (5.17), we obtain

$$
\begin{gathered}
E_{1, \min }=d_{\min }^{2} \frac{T}{4}+\frac{1}{2}\left[\left(d_{\min }^{2} \frac{T}{2}\right)^{2}+\frac{2 \pi^{2}\left(d_{\max }-d_{\min }\right)^{2}}{B_{1}^{2}}\right. \\
\left.\cdot\left\{\left(\frac{d_{\max }+d_{\min }}{2}\right)^{2}+\frac{1}{2}\left(\frac{d_{\max }-d_{\min }}{2}\right)^{2}-d_{\min }^{2}\right\}\right]^{1 / 2} .
\end{gathered}
$$

It can be verified that the optimum signal set $\mathscr{D}^{*}$ specified by (107)-(110) also satisfies the terminal constraint (103). Also, the signals in $\mathscr{D}^{*}$ satisfy the equidistance constraint 1 .
The greatest value $E_{1, \max }$ of $E_{1}$ for which $\mathscr{D}^{*}$ is optimal corresponds to the "full-width" PPM signal set with $\sigma_{2}=(T / 2 a$ $\left.-2 \sigma_{1}\right)$. Setting $\sigma_{2}=\left(T / 2 a-2 \sigma_{1}\right)$ and $E_{1}=E_{1, \max }$ in (5.17)(5.19), we obtain from

$$
E_{1, \max }=\int_{0}^{T / 2}\left\{d^{*}(t ; i)\right\}^{2} d t
$$

that

$$
\begin{aligned}
E_{1, \max }= & \frac{T}{2 a}\left[(a-1) d_{\min }^{2}+d_{\max }^{2}\right]-\frac{1}{E_{1, \max }} \frac{\pi^{2}\left(d_{\max }-d_{\min }\right)^{2}}{4 B_{1}^{2}} \\
& \cdot\left[d_{\max }^{2}-\left(\frac{d_{\max }+d_{\min }}{2}\right)^{2}-\frac{1}{2}\left(\frac{d_{\max }-d_{\min }}{2}\right)^{2}\right],
\end{aligned}
$$

which upon solving gives

$$
\begin{aligned}
E_{1, \max }= & \frac{T}{2 a}\left\{(a-1) d_{\min }^{2}+d_{\max }^{2}\right\} \\
& +\frac{1}{2}\left[\left\{\frac{T}{2 a}\left((a-1) d_{\min }^{2}+d_{\max }^{2}\right)\right\}^{2}\right. \\
& -\frac{2 \pi^{2}\left(d_{\max }-d_{\min }\right)^{2}}{4 B^{2}} \\
& \left.\cdot\left\{d_{\max }^{2}-\left(\frac{d_{\max }+d_{\min }}{2}\right)^{2}-\frac{1}{2}\left(\frac{d_{\max }-d_{\min }}{2}\right)^{2}\right\}\right]^{1 / 2}
\end{aligned}
$$

\section{REFERENCES}

[1] I. M. Wozencraft and R. S. Kennedy, "Modulation and demodulation for probabilistic coding," IEEE Trans. Inform. Theory, vol. IT-12, pp. 291-297, July 1966.

[2] J. L. Massey, "Coding and modulation in digital communications," in Proc. Int. Zurich Seminar on Digital Communications, Switzerland, Mar. 1974.

[3] D. L. Snyder and I. B. Rhodes, "Some implications of the cutoff-rate criterion for coded direct-detection optical communication systems," IEEE Trans. Inform. Theory, vol. IT-26, pp. 327-338, May 1980.

[4] P. Narayan and D. L. Snyder, "The two user cutoff rate region for an asynchronous and a synchronous multiple access channel are the same," IEEE Trans. Inform. Theory, vol. IT-27, pp. 414-419, July 1981.

[5] D. Slepian and J. K. Wolf, "A coding theorem for multiple access channels," Bell Syst. Tech. J., vol. 52, pp. 1937-1076, 1973.

[6] R. L. Peterson and D. J. Costello, Jr., "Error probability and free distance bounds for two-user tree codes on multiple-access channels," IEEE Trans. Inform. Theory, vol. IT-26, pp. 658-670, Nov. 1980.

[7] R. G. Gallager, Information Theory and Reliable Communication. New York: Wiley, 1968.

[8] M. Athans and F. C. Schweppe, "Optimal waveform design via control theoretic concepts," Inform. Contr., vol. 10, pp. 335-377, 1967.

[9] F. Amoroso, "The bandwidth of digital data signals," IEEE Commun. Magazine, vol. 18, pp. 13-24, Nov. 1980.

[10] J. M. Wozencraft and I. M. Jacobs, Principles of Communication Engineering. New York: Wiley, 1965.

[11] H. Sagan, Introduction to the Calculus of Variations. New York: McGraw-Hill, 1969.

[12] D. Slepian and H. O. Pollak, "Prolate spheroidal wave functions, fourier analysis and uncertainty-I," Bell Syst. Tech. J., vol. 40, pp. 43-84, Jan. 1961.

[13] M. Athans and P. L. Falb, Optimal Control. New York, McGraw-Hill, 1966.

[14] E. B. Lee and L. Markus, Foundations of Optimal Control Theory. New York: Wiley, 1967.

[15] D. Slepian, "Asymptotic expansions for prolate spheroidal wave functions," J. Math. Phys., vol. XLIV, pp. 99-140, June 1965. 